\title{
Mixing in manipulated turbulence
}

\author{
Arkadiusz K. Kuczaj(1) ${ }^{(1)}$ Bernard J. Geurts ${ }^{(1,2)}$
}

(1) Multiscale Modeling and Simulation, NACM, J.M. Burgers Center, Faculty EEMCS, University of Twente, P.O. Box 217, 7500 AE Enschede, The Netherlands

2) Anisotropic Turbulence, Fluid Dynamics Laboratory, Department of Applied Physics, Eindhoven University of Technology, P.O. Box 513, 5600 MB Eindhoven, The Netherlands

(October 8, 2018)

A' new computational framework for the simulation of turbulent flow through complex objects and jalong irregular boundaries is presented. This is motivated by the application of metal foams in com¿pact heat-transfer devices, or as catalyst substrates in process-engineering. The flow-consequences of - such complicated objects are incorporated by adding explicit multiscale forcing to the Navier-Stokes Sequations. The forcing represents the simultaneous agitation of a wide spectrum of length-scales when flow passes through the complex object. Two types of forcing procedures are investigated; With reference to the collection of forced modes these procedures are classified as 'constant-energy' Cor 'constant-energy-input-rate'. It is found that a considerable modulation of the traditional energy eascading can be introduced with a specific forcing strategy. In spectral space, forcing yields strongly localized deviations from the common Kolmogorov scaling law, directly associated with the explicitly forced scales. In addition, the accumulated effect of forcing induces a significant non-local alteration of the kinetic energy including the spectrum for the large scales. Consequently, 7 a manipulation of turbulent flow can be achieved over an extended range, well beyond the directly forced scales. Compared to flow forced in the large scales only, the energy in broad-band forced turbulence is found to be transferred more effectively to smaller scales. The turbulent mixing of a passive scalar field is also investigated, in order to quantify the physical-space modifications of transport processes in multiscale forced turbulence. The surface-area and wrinkling of level-sets of the scalar field are monitored as measures of the influence of explicit forcing on the local and global mixing efficiency. At small Schmidt numbers, the values of surface-area are mainly governed by the large scale sweeping-effect of the flow while the wrinkling is influenced mainly by the agitation of the smaller scales.

This paper is associated with the focus-issue Multi-scale Interactions in Turbulent Flows.

U.

\section{Introduction}

Various multiscale phenomena in turbulent flows arise from the passage of fluid through and along geometrically complex objects placed inside the flow-

To whom correspondence should be addressed (a.k.kuczaj@utwente.nl) 
domain. The corresponding perturbations of the flow arise simultaneously on a range of length-scales and find their origin in the complexity of the boundaries of these objects. A motivating example is the flow through a porous region such as a metal foam depicted in Fig. 1. Many more examples can readily be mentioned, arising in different technological applications or in numerous natural flows, including flow over forest canopies $[1,3,12]$.

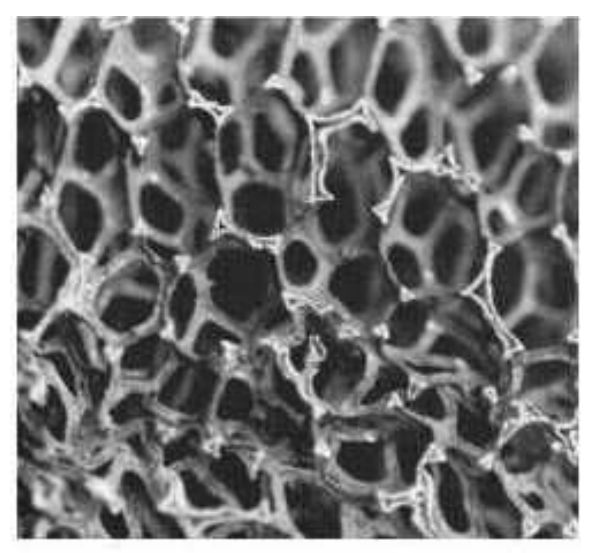

Figure 1. A porous nickel foam contains various geometrical complexities on different length-scales [29].

The purpose of this paper is to investigate the computational modeling of flows through complex regions via the introduction of explicit forcing terms in the Navier-Stokes equations. Consistent with the many shape-details of the obstructing objects, such forcing will need to represent the perturbation of the flow on various length-scales simultaneously. This distinguishes the proposed computational modeling from more conventional forced turbulence procedures. In the latter the flow agitation is restricted to a few large scales only with the aim to observe the development of a natural inertial range at smaller scales in the turbulent flow $[24,46]$. Instead, in this paper we allow the forcing of a collection of widely different modes. The consequences for transport and dispersion in such turbulent flows will be studied both in spectral - as well as in physical space. We will primarily establish the degree by which the spectral properties of a turbulent flow can be modified relative to the classical Kolmogorov scaling, and quantify the efficiency with which embedded scalar fields can be mixed by the modulated flow.

Complementary to the proposed explicit forcing approach, two alternative formulations have been put forward in literature to capture the flow in and around complex objects. These include the explicit boundary modeling [49] 
as well as an approximation in terms of effective boundary conditions and (surface) roughness parameters [20,22]. The roughness parameterization has been introduced for situations in which the roughness length-scales are much smaller than the boundary layer thickness [44]. For geometries which display both large- and small-scale contortions of the shape of the object, compared to the boundary layer thickness, the surface-roughness parametrization may not be sufficiently accurate [10]. Alternatively, in case of explicit boundary modeling, no-slip conditions are imposed at all the intricate shape-details of the object. This computational approach can in principle achieve full accuracy but is limited to cases of modest complexity in view of the elaborate geometric modeling and the high computational expenses that are required $[4,5]$.

Flow through complex gasket structures may give rise to self-similar turbulence spectra which do not follow the well known Kolmogorov -5/3 slope [27]. Such non-Kolmogorov turbulence was observed in flows over tree canopies, and is reminiscent of a spectral short-cut feature that was also observed experimentally [12]. In this paper we investigate the potential of multiscale forcing to accurately characterize such dynamic flow-consequences of complex domain boundaries without the need to explicitly account for their intricate geometrical shape. We consider the incompressible Navier-Stokes equations with multiscale forcing working as a stirrer whose dynamical effects are controlled by a distribution of simultaneously perturbed length-scales.

To arrive at a multiscale modeling that is quantitatively linked to actual complex objects several steps need to be taken. In this paper we address a first step in which we examine in some detail the influence different forcing procedures have on the energy dynamics in spectral space and the mixing characteristics in physical space. Special attention is devoted to the mixing efficiency of a passive tracer by monitoring the surface area and wrinkling of level-sets of these scalar fields [14]. Specifically we look at the instantaneous and accumulated effect on surface area and wrinkling caused by broad-band forcing.

Different divergence-free forcing procedures will be applied to directly perturb a large number of flow-scales. The alterations of the flow dynamics express themselves clearly in the kinetic energy. The transfer of energy toward smaller scales is found to increase considerably, compared to the case in which only large scales are forced. When a specific narrow band of scales is agitated by the forcing, then the locally higher spectral energy is not 'compatible' with the molecular dissipation-rate and an accelerated transfer is observed toward smaller scales. This effect is found for both families of forcing methods, i.e., constant-energy and constant-energy-input-rate. The kinetic energy spectrum is also modified non-locally, in a range of scales that are larger than the directly forced scales. Consequently the agitation of a band of small length-scale features can accumulate and also induce significant alterations of the largest 
flow-features, e.g., by contributing to an increased backscatter.

The changes in the flow-dynamics due to the application of broad-band forcing application also has consequences for the turbulent transport properties of the flow. This may be expressed in terms of the mixing efficiency of embedded passive scalars. In particular, monitoring the surface-area of level-sets of the passive scalar allows to characterize changes in the large-scale sweeping of the flow, due to the forcing. Likewise, the more localized motions directly affect the 'wrinkling' of the passive scalar level-sets. The dependence of these measures for the mixing efficiency on forcing parameters can be used to quantify the mixing efficiency arising from agitation of different bands of flow-structures with different forcing strengths. Specifically, we investigate the dispersion of strongly localized initial scalar concentrations. The direct numerical simulation of the forced turbulence shows that the maximal surface-area and wrinkling as well as the time at which such a maximum is achieved can be controlled by variation of forcing parameters. The time-integrated surface-area and wrinkling are indicators of the accumulated effect. The simulations show that at small Schmidt numbers, a higher emphasis on small-scale flow agitation yields a significant increase in the time-integrated total mixing of the flow.

The organization of this paper is as follows. In section 2 the simulation method, together with the explicit forcing strategies are introduced. Section 3 is devoted to the modulation of the cascading process associated with the different forcing methods. The consequences of forced turbulence for transport and dispersion in physical space will be quantified in section 4 . Concluding remarks are collected in section 5 .

\section{Simulation of forced turbulence}

In this section we will first introduce the governing equations (subsection 2.1) and subsequently describe the explicit forcing strategies that are used to drive the flow (subsection 2.2). Two types of deterministic forcing strategies will be included: procedures which yield constant-energy in the collection of forced modes, and procedures which correspond to a constant-energy-input-rate for these modes. Subsequently, the computational method, its validation and parallel performance will be described (subsection [2.3). 


\subsection{Governing equations}

The dimensionless system of nonlinear partial differential equations which governs the flow of a viscous incompressible fluid is given by:

$$
\left\{\begin{array}{l}
\frac{\partial \mathbf{v}(\mathbf{x}, t)}{\partial t}+(\mathbf{v}(\mathbf{x}, t) \cdot \nabla) \mathbf{v}(\mathbf{x}, t)=-\frac{1}{\rho} \nabla p(\mathbf{x}, t)+\nu \nabla^{2} \mathbf{v}(\mathbf{x}, t)+\mathbf{f}(\mathbf{x}, t) \\
\nabla \cdot \mathbf{v}(\mathbf{x}, t)=0
\end{array}\right.
$$

where $\mathbf{v}$ is the velocity field, $\rho$ is the constant mass-density and $p$ the pressure. The dimensionless viscosity is the inverse of the computational Reynolds number Re, i.e., $\nu=1 / \operatorname{Re}$, and $\mathbf{f}$ is the external forcing which we will specify in subsection 2.2. This system of equations may be rewritten in terms of the vorticity $\omega(\mathbf{x}, t)=\nabla \times \mathbf{v}(\mathbf{x}, t)$. Making use of the identity:

$$
(\mathbf{v}(\mathbf{x}, t) \cdot \nabla) \mathbf{v}(\mathbf{x}, t)=\omega(\mathbf{x}, t) \times \mathbf{v}(\mathbf{x}, t)+\frac{1}{2} \nabla\left(|\mathbf{v}(\mathbf{x}, t)|^{2}\right)
$$

we may express (11) as:

$$
\left(\frac{\partial}{\partial t}-\nu \nabla^{2}\right) \mathbf{v}(\mathbf{x}, t)=\mathbf{w}(\mathbf{x}, t)-\nabla\left(\frac{1}{\rho} p(\mathbf{x}, t)+\frac{1}{2}|\mathbf{v}(\mathbf{x}, t)|^{2}\right)+\mathbf{f}(\mathbf{x}, t)
$$

where we introduced the nonlinear term $\mathbf{w}(\mathbf{x}, t)=\mathbf{v}(\mathbf{x}, t) \times \omega(\mathbf{x}, t)$.

The flow domain is assumed to be periodic with the same period in each of the three coordinate directions. An efficient representation of the solution in terms of Fourier modes can be adopted $[6,33,52]$ in which the velocity $\mathbf{v}(\mathbf{x}, t)$ is expanded as:

$$
\mathbf{v}(\mathbf{x}, t)=\sum_{\mathbf{k}} \mathbf{u}(\mathbf{k}, t) e^{\imath \mathbf{k} \cdot \mathbf{x}}
$$

and the wavevector $\mathbf{k}(k=|\mathbf{k}|)$ has components $k_{\alpha}=2 \pi n_{\alpha} / L_{b}$, $n_{\alpha}=0, \pm 1, \pm 2, \ldots$ for $\alpha=1,2,3$. The dimensionless length of the periodic domain is denoted by $L_{b}$ and $u_{\alpha}(\mathbf{k}, t)$ is the Fourier-coefficient corresponding to the $\mathbf{k}$-th mode of $v_{\alpha}(\mathbf{x}, t)$. The equation governing the evolution of the Fourier-coefficients is given by:

$$
\left(\frac{\partial}{\partial t}+\nu k^{2}\right) \mathbf{u}(\mathbf{k}, t)=\mathbf{W}(\mathbf{k}, t)-\imath \mathbf{k} \mathcal{F}\left(\frac{1}{\rho} p(\mathbf{x}, t)+\frac{1}{2}|\mathbf{v}(\mathbf{x}, t)|^{2}, \mathbf{k}\right)+\mathbf{F}(\mathbf{k}, t)
$$

where $\mathcal{F}(a(\mathbf{x}, t), \mathbf{k})$ denotes the Fourier-coefficient of the function $a(\mathbf{x}, t)$ cor- 
responding to wavevector $\mathbf{k}$ :

$$
\mathcal{F}(a(\mathbf{x}, t), \mathbf{k})=A(\mathbf{k}, t) \quad \text { if } \quad a(\mathbf{x}, t)=\sum_{\mathbf{k}} A(\mathbf{k}, t) e^{\imath \mathbf{k} \cdot \mathbf{x}}
$$

In addition, $\mathbf{W}(\mathbf{k}, t)$ and $\mathbf{F}(\mathbf{k}, t)$ denote the $\mathbf{k}$-th Fourier-coefficient of the nonlinearity $\mathbf{w}(\mathbf{x}, t)$ and forcing $\mathbf{f}(\mathbf{x}, t)$, respectively.

In spectral space the pressure term may be eliminated from (5) if use is made of the incompressibility condition. This is equivalent to the well-known practice of solving a Poisson equation for the pressure in physical space formulations [48]. If we multiply (5) by $\mathbf{k}$, use the continuity equation in spectral space, i.e., $\mathbf{k} \cdot \mathbf{u}(\mathbf{k}, t)=0$, and assume that the forcing itself is divergence-free, so that $\mathbf{k} \cdot \mathbf{F}(\mathbf{k}, t)=0$, the pressure term can be written as:

$$
\mathcal{F}\left(\frac{1}{\rho} p(\mathbf{x}, t)+\frac{1}{2}|\mathbf{v}(\mathbf{x}, t)|^{2}, \mathbf{k}\right)=\frac{\mathbf{k} \cdot \mathbf{W}(\mathbf{k}, t)}{\imath k^{2}}
$$

The equation for the Fourier-coefficients of the velocity field (5) may now be written as:

$$
\left(\frac{\partial}{\partial t}+\nu k^{2}\right) \mathbf{u}(\mathbf{k}, t)=\mathbf{W}(\mathbf{k}, t)-\mathbf{k}\left(\frac{\mathbf{k} \cdot \mathbf{W}(\mathbf{k}, t)}{k^{2}}\right)+\mathbf{F}(\mathbf{k}, t)
$$

This may be expressed in a more compact form in terms of the projection operator $\mathbf{D}$ defined by:

$$
D_{\alpha \beta}=\delta_{\alpha \beta}-\frac{k_{\alpha} k_{\beta}}{k^{2}}
$$

This operator restricts the solution to the space of divergence-free fields, represented by Fourier-coefficients $\mathbf{u}(\mathbf{k}, t)$ which lie in the plane normal to the wavevector $\mathbf{k}$. We obtain the governing equation for the desired Fouriercoefficients as:

$$
\left(\frac{\partial}{\partial t}+\nu k^{2}\right) \mathbf{u}(\mathbf{k}, t)=\mathbf{D W}(\mathbf{k}, t)+\mathbf{F}(\mathbf{k}, t)
$$

A more detailed discussion of this spectral approach to the Navier-Stokes equations is available in [33]. It forms the basis for the numerical treatment that will be specified in subsection 2.3 .

In various applications the dispersion of a passive scalar by a turbulent flow is of central importance. Passive scalar transport may be used to characterize the physical space consequences of multiscale forced turbulence. The governing 
equation for the evolution of the scalar concentration $C(\mathbf{x}, t)$ contains advection by the velocity field $\mathbf{v}(\mathbf{x}, t)$ as well as diffusion. In physical space this may be expressed as:

$$
\frac{\partial C(\mathbf{x}, t)}{\partial t}+(\mathbf{v}(\mathbf{x}, t) \cdot \nabla) C(\mathbf{x}, t)=\kappa \nabla^{2} C(\mathbf{x}, t)
$$

where $\kappa$ is the non-dimensional molecular diffusivity of the scalar. Compared to the dimensionless viscosity in (11) we adopt $\kappa=\nu / \mathrm{Sc}$ where the Schmidt number Sc characterizes the scalar diffusion. Roughly speaking, if Sc $>1$ then the scalar field displays a wider range of dynamically important length-scales, compared to the turbulent velocity field, while values $\mathrm{Sc}<1$ indicate a comparably smoother scalar field. The equation which governs the development of the Fourier-coefficients $c(\mathbf{k}, t)$ of the scalar field $C(\mathbf{x}, t)$ can readily be found as:

$\left(\frac{\partial}{\partial t}+\kappa k^{2}\right) c(\mathbf{k}, t)=Z(\mathbf{k}, t) \quad$ where $\quad Z(\mathbf{k}, t)=\mathcal{F}((\mathbf{v}(\mathbf{x}, t) \cdot \nabla) C(\mathbf{x}, t), \mathbf{k})$

The changes in the turbulent transport properties of the flow due to the multiscale forcing can be investigated by considering the evolution of the scalar concentration at different Schmidt numbers. The structure of the left-hand side of (12) is identical to the Navier-Stokes equations in (10). This allows to adopt the same time-stepping method, as will be specified in subsection 2.3 .

To quantify the spectral-space effect of multiscale forcing, and also to be able to concisely formulate the different forcing procedures in the next subsection, we consider the kinetic energy. The equations which govern the Fourier coefficients (10) can be written in index notation as:

$$
\left(\frac{\partial}{\partial t}+\nu k^{2}\right) u_{\alpha}(\mathbf{k}, t)=\Psi_{\alpha}(\mathbf{k}, t)+F_{\alpha}(\mathbf{k}, t)
$$

where $\Psi_{\alpha}(\mathbf{k}, t)=D_{\alpha \beta} W_{\beta}(\mathbf{k}, t)$ is the nonlinear term. Multiplying this equation by the complex-conjugate $u_{\alpha}^{*}(\mathbf{k}, t)$ and summing over the three coordinate directions, we obtain the kinetic energy equation:

$$
\left(\frac{\partial}{\partial t}+2 \nu k^{2}\right) E(\mathbf{k}, t)=u_{\alpha}^{*}(\mathbf{k}, t) \Psi_{\alpha}(\mathbf{k}, t)+u_{\alpha}^{*}(\mathbf{k}, t) F_{\alpha}(\mathbf{k}, t)
$$

where $E(\mathbf{k}, t)=\frac{1}{2}|\mathbf{u}(\mathbf{k}, t)|^{2}$ is the kinetic energy in mode $\mathbf{k}$. Introducing the notation for the rate of energy transfer $T(\mathbf{k}, t)=u_{\alpha}^{*}(\mathbf{k}, t) \Psi_{\alpha}(\mathbf{k}, t)$, the rate of energy injection by the forcing $T_{F}(\mathbf{k}, t)=u_{\alpha}^{*}(\mathbf{k}, t) F_{\alpha}(\mathbf{k}, t)$ and the energy dis- 
sipation rate $\varepsilon(\mathbf{k}, t)=2 \nu k^{2} E(\mathbf{k}, t)$, we can write (14) equation as:

$$
\frac{\partial E(\mathbf{k}, t)}{\partial t}=-\varepsilon(\mathbf{k}, t)+T(\mathbf{k}, t)+T_{F}(\mathbf{k}, t)
$$

This formulation clarifies that the rate of change of kinetic energy $E(\mathbf{k}, t)$ is connected with dissipation, expressed by the viscous term $\varepsilon(\mathbf{k}, t)$, with transfer to/from different wavenumbers, expressed by $T(\mathbf{k}, t)$, and with the forcing term $T_{F}(\mathbf{k}, t)$.

The different contributions to the rate of change of the kinetic energy typically act in distinct wavenumber regions. The forcing term $T_{F}(\mathbf{k}, t)$ is non-zero in the forced modes only. In this paper the collection of forced modes will always contain a low wavenumber band corresponding to large-scale forcing of the flow. In addition, possible higher wavenumber contributions can be included in $T_{F}(\mathbf{k}, t)$. In contrast, energy dissipation $\varepsilon(\mathbf{k}, t)$ is defined in the entire spectral space, but it is dynamically important primarily for the high wavenumber range, i.e., acting on structures below the dissipation lengthscale. Finally, the transfer term $T(\mathbf{k}, t)$ is basic to the development of an energy cascade and is a dominant contribution for wavenumbers in an inertial range [33]. In the multiscale forcing cases, we will also introduce forcing generally in the same region as where the transfer $T(\mathbf{k}, t)$ is dynamically important. Hence, the effects of the multiscale forcing relate directly to the 'competition' between the dynamics introduced by the forcing procedure and the 'natural' transfer of energy to other modes in the spectrum.

In the formulation of forcing procedures and in the evaluation of the kinetic energy dynamics, one frequently adopts shell-averaging. The basic operation consists of averaging over spherical shells of thickness $2 \pi / L_{b}$ centered around the origin. The $n$-th spherical shell is given by $\frac{2 \pi}{L_{b}}(n-1 / 2)<|\mathbf{k}| \leq \frac{2 \pi}{L_{b}}(n+1 / 2)$ and will be denoted by $\mathbb{K}_{n}$. Applying shell-averaging to a function $h(\mathbf{k}, t)$ defined in spectral space we obtain:

$$
\bar{h}(n, t)=\frac{1}{P_{n}} \sum_{\mathbb{K}_{n}} h(\mathbf{k}, t) \quad ; \quad P_{n}=\sum_{\mathbb{K}_{n}} 1
$$

where $P_{n}$ is the number of modes in the $n$-th shell. Applying the shell-averaging (16) to the energy equation (15) we end up with:

$$
\frac{\partial \bar{E}(n, t)}{\partial t}=-\bar{\varepsilon}(n, t)+\bar{T}(n, t)+\bar{T}_{F}(n, t)
$$

which indicates that the interpretation of the various contributions to the rate of change of the kinetic energy at mode $\mathbf{k}$ also applies to the shell-averaged 
formulation. In literature it is common to introduce a numerical correction factor when averaging over shells. This is used to compensate for the nonuniform distribution of modes within the discrete spherical shells $[11,26]$. We will follow the convention used in $[11,51,52]$ when presenting the energy-spectra. This implies that we multiply $\bar{h}(n, t)$ by a factor $4 \pi n^{2}$ which is associated with the 'expected number modes' within the discrete shell. The definition of the energy spectrum that we will adopt is given by $E_{n}=\left(4 \pi n^{2} / P_{n}\right) \sum_{\mathbb{K}_{n}} E(\mathbf{k}, t)$. Finally, summing (15) over all wavevectors $\mathbf{k}$ or, equivalently, (17) over all shells yields the evolution equation for the total energy in the system:

$$
\frac{d \widehat{E}(t)}{d t}=-\widehat{\varepsilon}(t)+\widehat{T}_{F}(t) \quad ; \quad \widehat{h}(t)=\sum_{n} P_{n} \bar{h}(n, t)=\sum_{\mathbf{k}} h(\mathbf{k}, t)
$$

where use was made of the fact that the contribution of the transfer term $T(\mathbf{k}, t)$ is such that it only re-distributes energy over the various modes, which implies that its sum over all wavenumbers $\widehat{T}(t)=0$.

Next to spherical shells, it is convenient to introduce spherical wavenumber bands which consist of several adjacent shells. We denote the wavenumber band which consists of $\frac{2 \pi}{L_{b}}(m-1 / 2)<|\mathbf{k}| \leq \frac{2 \pi}{L_{b}}(p+1 / 2)$ by $\mathbb{K}_{m, p}$, where $m \leq p$. The corresponding average over $\mathbb{K}_{m, p}$ of a function $h(\mathbf{k}, t)$ is given by:

$$
\widetilde{h}^{(m, p)}(t)=\frac{1}{P_{m, p}} \sum_{n=m}^{p} P_{n} \bar{h}(n, t)=\frac{1}{P_{m, p}} \sum_{\mathbb{K}_{m, p}} h(\mathbf{k}, t) \quad ; \quad P_{m, p}=\sum_{n=m}^{p} P_{n}
$$

To complete the computational model, we will next introduce the explicit forcing strategies that will be investigated in this paper.

\section{$2.2 \quad$ Explicit forcing procedures}

Forced turbulence in a periodic box is one of the most basic numerically simulated turbulent flows. It is achieved by applying large-scale forcing to the Navier-Stokes equations. As a result, at sufficiently high Reynolds number the well-known turbulent cascade develops in an inertial range of scales which are much smaller than the length-scale of the forced modes $[27,28,33]$. The statistical equilibrium that is reached is characterized by a balance between the input of energy through the large-scale forcing and the viscous dissipation at scales beyond the Kolmogorov dissipation scale.

Various forcing procedures have been proposed in literature. Generally, if the forcing is restricted to large scales only, the specific details of the procedure do not have such a large effect on the properties of the developing inertial range at sufficiently finer scales. However, since we wish to extend the forcing 
to act on a wide range of scales simultaneously, including parts of an inertial sub-range, the differences between alternative forcing procedures become more pronounced. Investigating these differences is an essential step toward quantitative modeling of flow through complex gasket structures and forms the main focus of this paper. In this subsection, we will recover the definition and some of the motivation for several characteristic forcing procedures.

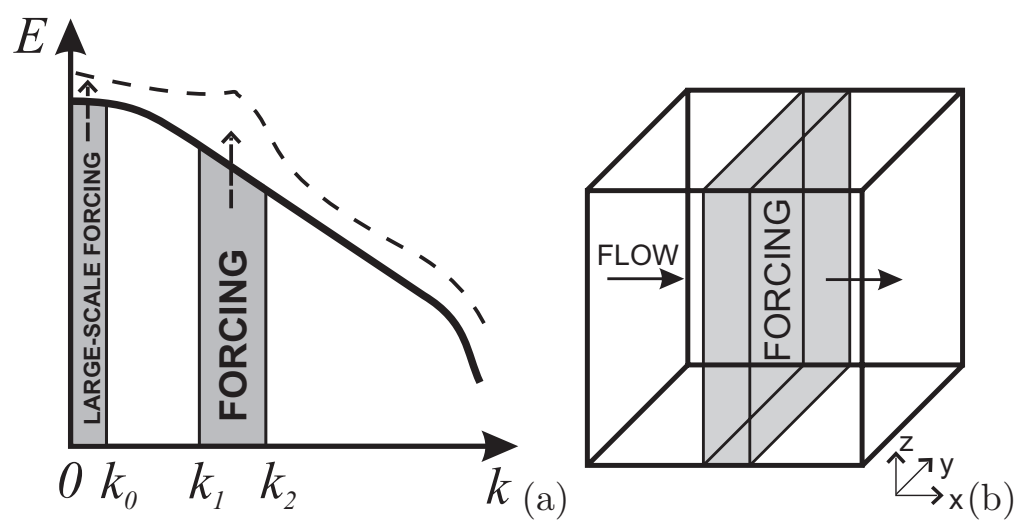

Figure 2. Definition of two-band forcing in spectral space (a) and localization of forcing within a slab in physical space (b).

In multiscale forcing, the flow is agitated over a wide range of modes. To investigate the effects of such forcing we will focus on cases in which one additional spherical band of scales is forced, next to the common forcing of the large scales. We consider the general situation as depicted in Fig. 2(a). The large scales are in the range $k \leq k_{0}$ and an additional band of small scales is defined by $k_{1}<k \leq k_{2}$. The forcing method can also incorporate cases in which only part of the domain is occupied by a complex obstruction, as sketched for the case of a slab in Fig. 2(b). In fact, by introducing an 'indicator' function $\Theta(\mathbf{x}, t)$ to locate the complex object within the flow domain $(\Theta=0$ outside the region occupied by the object and 1 elsewhere), the forcing can accommodate such spatial localization in a flexible manner. In spectral space, the introduction of $\Theta(\mathbf{x}, t)$ implies that the forcing term in spectral space is represented by the convolution product of the actual forcing $F(\mathbf{k}, t)$ and the Fourier-transform of the indicator function. However, in the present paper such complications will not be included and we will only consider forcing procedures applied in the entire physical domain.

Forcing procedures may be classified in different ways. We first distinguish forcing schemes which keep the total energy in the collection of forced modes identical to its value in the initial condition. This will be referred to as class ' $\mathrm{A}$ ' forcing procedures. Next, we identify forcing schemes which are characterized by a constant energy input rate, introduced via the collection of forced modes. 
This group will be referred to as class 'B'. In either class of schemes, the flows develop around a well defined statistically stationary state, but time-dependent variations in the total energy and in the energy input rate may occur.

Apart from a distinction concerning the way energy is introduced into the flow, one may classify forcing schemes as 'deterministic' or 'stochastic'. Stochastic forcing schemes may introduce an element of uncorrelated randomness, e.g., by restricting the forcing to a random subset of the collection of forced modes every time the forcing is invoked. However, the primary question of locality of the modulation of the energy spectrum can be addressed more directly using deterministic schemes and in this paper we will restrict to these procedures. We next introduce some characteristic forcing schemes in either class 'A' or class 'B'.

Class ' $A$ ': constant-energy forcing. Various methods can be formulated which are such that the kinetic energy in the forced modes remains constant. The simplest possibility arises by requiring that $u_{\alpha}(\mathbf{k}, t)$ itself remains constant for all $\mathbf{k}$ in the collection of forced modes. This was first proposed in [43] and implies for the forcing in spectral space:

$$
\text { A1 : } \quad F_{\alpha}(\mathbf{k}, t)=\nu k^{2} u_{\alpha}(\mathbf{k}, t)-\Psi_{\alpha}(\mathbf{k}, t)
$$

One readily verifies, using (13), that $\partial_{t} u_{\alpha}(\mathbf{k}, t)=0$ and in particular this implies that $\partial_{t} E(\mathbf{k}, t)=0$ for each of the forced modes. Hence, also the total kinetic energy contained in all the forced modes stays constant in time. The energy input rate corresponding to (20) is given by $T_{F}(\mathbf{k}, t)=\varepsilon(\mathbf{k}, t)-T(\mathbf{k}, t)$ for each of the forced modes. This input rate may vary considerably in time, as the unsteady flow will lead to a strong time-dependence of the energy transfer $T$ for the forced modes.

The basic method (20) has motivated the formulation of a number of extensions which relax the requirement that the Fourier-coefficient is strictly constant. In [8] the method was modified to require that $|\mathbf{u}(\mathbf{k}, t)|=$ const, i.e., equal to its initial value, for the forced modes. This allows for the possibility that the phases of the Fourier-coefficients may evolve in time. The corresponding forcing is given by:

$$
F_{\alpha}(\mathbf{k}, t)=\left(\nu k^{2}-\frac{T(\mathbf{k}, t)}{2 E(\mathbf{k}, t)}\right) u_{\alpha}(\mathbf{k}, t)=(\varepsilon(\mathbf{k}, t)-T(\mathbf{k}, t)) \frac{u_{\alpha}(\mathbf{k}, t)}{2 E(\mathbf{k}, t)}
$$

One may readily verify that this implies $\partial_{t} E(\mathbf{k}, t)=0$ for the forced modes. Forcing expressed in (20) or (21) was found to yield quite large fluctuations in the energy input rate [45]. 
Typically, the forced modes are ordered according to the wavenumber shell to which they belong. A shell-oriented simplification of (21) was proposed [25,26]:

$$
F_{\alpha}(\mathbf{k}, t)=(\bar{\varepsilon}(k, t)-\bar{T}(k, t)) \frac{u_{\alpha}(\mathbf{k}, t)}{2 E(\mathbf{k}, t)}
$$

This forcing also preserves the total kinetic energy in the forced modes. The three forcing procedures (20), (21) and (22) are quite comparable, both in terms of their fluid-physics motivation and in terms of their turbulent flow predictions. Therefore, we will only present actual simulation results obtained with (20), which are quantitatively representative for the other two forcing procedures in this group.

The forcing methods described so far preserve the kinetic energy that is contained in the collection of forced modes. However, considerable variations in the total energy in the system can still arise. The reverse can also be realized, i.e., forced turbulence in which the total kinetic energy in the system is constant, but the energy in different modes may vary in time. For this purpose, the forcing should not be formulated in terms of quantities related to individual modes or shell-averaged values, but rather contain averages over all modes $[18,30]$. The case of forcing in a single shell with $P$ modes can readily be specified. Specifically, if we replace the shell-average $\overline{(\cdot)}$ in the amplitude factor in (22) by the average over all modes $\widehat{(\cdot)}$ and use the fact that $\widehat{T}=0$, we obtain the forcing

$$
\mathrm{A} 2: \quad F_{\alpha}(\mathbf{k}, t)=\frac{\widehat{\varepsilon}(t)}{P} \frac{u_{\alpha}(\mathbf{k}, t)}{2 E(\mathbf{k}, t)}
$$

The A2-forcing implies an energy input rate $\widehat{T}_{F}=\widehat{\varepsilon}(t)$ and thus by (18) $d \widehat{E} / d t=0$. This method corresponds exactly to the negative viscosity procedure used to maintain quasi-steady turbulence direct numerical simulations results reported in $[21,23,24,50]$. Extension of A2-forcing to multiple shells can be realized in a number of ways. This will be described in more detail momentarily. A2-forcing will be compared to A1-forcing in the next section.

Class ' $B$ ': constant-energy-input-rate forcing. Next to forcing methods that can be associated with constant-energy, one may define forcing procedures in which the total energy input rate $\widehat{T}_{F}$ is constant. We first present such forcing methods with reference to a single band of forced modes. The way in which the energy input is distributed over several bands will be specified afterwards. 
A central example in the class of constant-energy-input-rate forcing methods was presented in [16]. Changing $\widehat{\varepsilon}(t)$ in (23) into the constant energy inputrate $\varepsilon_{w}$, the corresponding forcing term may be written as

$$
\mathrm{B} 1: \quad F_{\alpha}(\mathbf{k}, t)=\frac{\varepsilon_{w}}{P} \frac{u_{\alpha}(\mathbf{k}, t)}{2 E(\mathbf{k}, t)}
$$

The energy input-rate is found to be $\widehat{T}_{F}(t)=\varepsilon_{w}$, as desired by construction. The total energy in the system is no longer constant but governed by $d \widehat{E}(t) / d t=-\widehat{\varepsilon}(t)+\varepsilon_{w}$ which implies that the statistically stationary state that develops will show a dissipation rate that fluctuates about $\varepsilon_{w}$. This type of forcing was also studied in $[7,36,52]$. Further extensions of the basic forcing procedure (24) can be proposed in which an extra factor $k^{-q} ; q>0$ arises in the definition of $F_{\alpha}$. Such an extra factor implies that the forcing of higher wavenumber shells can be made to correspond to a specific shape (usually $k^{-5 / 3}$ to more directly 'impose' Kolmogorov turbulence). These forcing procedures will not be considered in this paper; for further details see [9,37].

Similar to A-forcing methods, one may formulate related procedures which are defined in terms of shell-averaged quantities. For example, analogous to (22), we may replace $E(\mathbf{k}, t)$ in (24) by $\bar{E}(n, t)$ to define the forcing of modes in the $n$-th shell. This type of forcing was found to yield basically the same results as those based on (24) and will not be presented explicitly in the rest of this paper.

The final forcing procedure that we will include in this paper was proposed recently in [32]. It was motivated as a model of flow through a fractal gasket which functions as a multiscale stirrer. This particular forcing may be associated with a constant energy input rate for the entire system. We modify the original forcing procedure slightly and considered in particular

$$
\text { B2 : } \quad F_{\alpha}(\mathbf{k}, t)=\frac{\varepsilon_{w} k^{\beta}}{\sum_{\mathbf{k} \in \mathbb{K}} \sqrt{2 E(\mathbf{k}, t)} k^{\beta}} e_{\alpha}(\mathbf{k}, t)
$$

where $\mathbb{K}$ denotes the set of forced modes. In this formulation, the complexity of the object is parameterized by the exponent $\beta$ which is related to the fractal dimension $D_{f}$ of the object through $\beta=D_{f}-2$. The vector $\mathbf{e}(\mathbf{k}, t)$ has the form:

$$
\mathbf{e}(\mathbf{k}, t)=\frac{\mathbf{u}(\mathbf{k}, t)}{|\mathbf{u}(\mathbf{k}, t)|}+\imath \frac{\mathbf{k} \times \mathbf{u}(\mathbf{k}, t)}{|\mathbf{k}||\mathbf{u}(\mathbf{k}, t)|}
$$

which contains a part in the direction of $\mathbf{u}$ and a part that is perpendicular to $\mathbf{u}$. 
Since $u_{\alpha}^{*} e_{\alpha}=|\mathbf{u}|=\sqrt{2 E(\mathbf{k}, t)}$ we find for the energy input rate:

$$
T_{F}(\mathbf{k}, t)=\varepsilon_{w} \frac{k^{\beta} \sqrt{2 E(\mathbf{k}, t)}}{\sum_{\mathbf{k} \in \mathbb{K}} k^{\beta} \sqrt{2 E(\mathbf{k}, t)}}
$$

In contrast to B1-forcing in which the energy input rate is constant in time for each of the forced modes separately, this 'fractal forcing' procedure only implies a constant energy input rate for the entire system. In fact, after summation over all forced modes the total energy input rate is found to be equal to $\widehat{T}_{F}(t)=\varepsilon_{w}$. Correspondingly, we find for the evolution of the total kinetic energy $d \widehat{E} / d t=-\widehat{\varepsilon}(t)+\varepsilon_{w}$, i.e., identical as obtained before for B1-forcing. In the original formulation in [32] the energy input rate $\varepsilon_{w}$ was replaced by the total dissipation rate $\widehat{\varepsilon}(t)$, which implies that $\widehat{E}$ is constant in time.

So far, the B1- and B2-forcing methods were defined with reference to a single band of modes. This band was assumed to contain $P$ modes and was identified by $\mathbb{K}$. The total energy input rate $\varepsilon_{w}$ was available to this band. In case more bands are forced simultaneously, the way the energy input-rate is divided over the individual bands, and among the modes within each band, needs to be specified. For two forced bands $\mathbb{K}_{m_{1}, p_{1}}$ and $\mathbb{K}_{m_{2}, p_{2}}$ with $P_{m_{1}, p_{1}}$ and $P_{m_{2}, p_{2}}$ modes respectively, such a partitioning involves two steps. First, a fraction $\varepsilon_{w, 1}=a \varepsilon_{w}$ of the total energy input-rate is 'allocated' to the first band and the remainder $\varepsilon_{w, 2}=(1-a) \varepsilon_{w}$ is used in the forcing of the second band $(0 \leq a \leq 1)$. Second, we divide the energy input-rate that is available for each band equally over all modes in the corresponding band. As an example, two-band B1-forcing may be defined as

$$
\text { B1 : } \begin{aligned}
F_{\alpha}(\mathbf{k}, t) & =\frac{a \varepsilon_{w}}{P_{m_{1}, p_{1}}} \frac{u_{\alpha}(\mathbf{k}, t)}{2 E(\mathbf{k}, t)} ; \quad k \leq k_{0} \\
& =\frac{(1-a) \varepsilon_{w}}{P_{m_{2}, p_{2}}} \frac{u_{\alpha}(\mathbf{k}, t)}{2 E(\mathbf{k}, t)} \quad ; \quad k_{1}<k \leq k_{2} \\
& =0 \quad ; \quad \text { otherwise }
\end{aligned}
$$

The two-band formulation of B2-forcing can be specified analogously, replacing $\varepsilon_{w}$ by either $a \varepsilon_{w}$ or $(1-a) \varepsilon_{w}$ and $\mathbb{K}$ by $\mathbb{K}_{m_{1}, p_{1}}$ or $\mathbb{K}_{m_{2}, p_{2}}$, respectively. Extending A2-forcing to more bands can be done in a similar way in which a fraction $a \widehat{\varepsilon}(t)$ is associated with the large-scale band and the remainder with the second band. The specific choice of $P_{m_{1}, p_{1}}$ and $P_{m_{2}, p_{2}}$ above implies that the energy is equally distributed between all modes within a forced band. We can go one step further and require the equal distribution of $\varepsilon_{w}$ over the forced shells contained in the bands. This implies changing $P_{m_{1}, p_{1}}$ and $P_{m_{2}, p_{2}}$ into the 
number of modes $P_{n}$ for each forced shell. Extension to more forced bands can be formulated analogously.

In the next subsection the numerical method will be specified in some detail, to complete the description of the physical and computational modeling used in this paper.

\subsection{Computational method and parallelization}

In this subsection we first specify the time-stepping method, then sketch some aspects of the implementation and subsequently describe the validation of the method.

Time evolution. To simulate the spectral solution governed by equations (10) and (12) we first rewrite these equations in a more general form having in mind that the evolution due to the diffusive terms can be computed exactly by introducing integrating factors $e^{\nu k^{2} t}$ and $e^{\kappa k^{2} t}$, respectively [6]. In fact, (10) and (12) may be expressed as:

$$
\frac{\partial \mathbf{U}(\mathbf{k}, t)}{\partial t}=\mathbf{G}(\mathbf{U}(\mathbf{k}, t))
$$

where

$$
\mathbf{U}=\left[\begin{array}{c}
\mathbf{u}(\mathbf{k}, t) e^{\nu k^{2} t} \\
c(\mathbf{k}, t) e^{\kappa k^{2} t}
\end{array}\right] \quad ; \quad \mathbf{G}=\left[\begin{array}{c}
(\mathbf{D W}(\mathbf{k}, t)+\mathbf{F}(\mathbf{k}, t)) e^{\nu k^{2} t} \\
Z(\mathbf{k}, t) e^{\kappa k^{2} t}
\end{array}\right]
$$

We use a constant time-step $\Delta t$ to obtain the solution at times $t_{n}=t_{0}+n \Delta t$. A four-stage, second-order, compact-storage Runge-Kutta method was implemented. The advancement of the solution over a full time-step requires four steps of the form:

$$
\mathbf{U}\left(\mathbf{k}, t_{n+\gamma}\right)=\mathbf{U}\left(\mathbf{k}, t_{n}\right)+\gamma \Delta t \mathbf{G}\left(\mathbf{U}\left(\mathbf{k}, t_{n+\xi}\right)\right)
$$

The intermediate solutions in the different stages can be found as follows. In stage 1 we adopt $(\gamma, \xi)=(1 / 4,0)$, stage 2 requires $(\gamma, \xi)=(1 / 3,1 / 4)$, stage 3 uses $(\gamma, \xi)=(1 / 2,1 / 3)$ and stage 4 completes the step with $(\gamma, \xi)=$ $(1,1 / 2)[15]$.

We consider turbulence in a cubic box of side $L_{b}$ with periodic boundary conditions and assume that the flow is statistically isotropic which implies that we require the same resolution in each coordinate direction. The direct numerical simulations will employ a resolution of $N^{3}$, where $N$ is the number of spectral-space grid-points that is used in each direction. This restricts 
the set of wavenumbers to $n_{\alpha}=0, \pm 1, \pm 2, \ldots, \pm(N / 2-1),-N / 2$. The cutoff wavenumber is given by $k_{\max }=\pi N / L_{b}$. In physical space this corresponds to a uniform grid $x_{\alpha}=j L_{b} / N$, where $j=0,1,2, \ldots, N-1$ in each coordinate direction.

We use the pseudo-spectral discretization method, i.e., the spatial derivative terms in the Navier-Stokes and passive scalar equations are computed via simple multiplications in the spectral space. The nonlinear terms in the equations are evaluated in physical space to avoid the evaluation of several computationally intensive convolution sums [6]. This procedure requires three steps. First, the Fourier-coefficients $\mathbf{u}(\mathbf{k}, t)$ and $c(\mathbf{k}, t)$ are used to obtain the velocity and scalar fields in the physical space. Subsequently, the velocity-velocity products and the velocity-scalar products are determined in physical space and finally the associated Fourier-coefficients of these products are obtained.

The finite resolution may give rise to well-known aliasing errors. In fact, the product of two Fourier-series based on a resolution with $N$ points gives rise to more small-scale modes than can be supported by the grid. As a result, these contributions can appear on the $N$-point resolution as seemingly lower wave-number modes. A detailed discussion of techniques allowing the partial or full removal of the aliasing error can be found in [6]. Several of these methods were tested, as described in the appendix, closely following [40,41]. The method of two shifted grids and spherical truncation was used in actual simulations. This removes the aliasing error completely which was found to be essential, especially to maintain the characteristics of the small turbulent scales.

Data decomposition and fast Fourier transforms. The simulation software was implemented in Fortran 90 and parallelized based on the framework given in [52] using the Message Passing Interface (MPI) [38]. Data are stored using the Hierarchical Data Format (HDF5) [19] which is a file format and library designed for scientific data-storage and handling. The choice of HDF5 was motivated by the flexible data-exchange between different platforms and its support of parallel I/O. High performance computations were done at SARA Computing and Networking Services (Amsterdam) on Silicon Graphics (SGI) Altix 3700 and Origin 3800 CC-NUMA systems (for more details see [42]).

The critical performance factors in the parallel implementation of the pseudo-spectral discretization method are the domain decomposition and the algorithm for the three-dimensional Fast Fourier Transform (FFT). These two implementation decisions are essential since they determine almost all aspects of the data-exchange between domains and most of the floating point operations. It is important to obtain a data decomposition which permits for fast transfer of data between processors. To obtain parallel Fourier transforms we adopted procedures from two libraries: SCSL [17] and FFTW [13]. 
Moreover, since access to memory and the number and speed of available CPU-s may differ considerably among different computational platforms, significant improvements in the processing time can be achieved by platformdependent optimization.

The speedup of the parallel implementation was checked by simulating decaying turbulence at a resolution of $256^{3}$. A time-interval $0 \leq t \leq 0.05$ was considered. This case corresponds to 28 time-steps with 5 data evaluation and reporting stages. In a non-dedicated SGI Altix 3700 environment we obtained on $4,8,16,32,64$ processors the following speedup numbers: $3.9,7.5,14,26,47$, respectively. The best performance results were obtained by a cache-unfriendly parallelization along the second array dimension. This gives the opportunity of minimal data exchange and reshuffling between processors and illustrates that the speed of the processors overwhelms the abilities of direct access to the memory. This was found to be the critical issue for the hardware that was available.

Code validation. To validate the implementation of the pseudo-spectral method, decaying homogeneous isotropic turbulence was simulated at two different Reynolds numbers. The initial condition was taken from [35], which was generated on the basis of the Pao spectrum [39]. For further details we refer to [34]. This flow was studied extensively using high-order finite-volume discretization and explicit Runge-Kutta time-stepping. Special attention was given to the degree of convergence that could be achieved using the finite volume approach. These data provide a clear point of reference with which the present pseudo-spectral flow-solver can be compared.

\begin{tabular}{c|c|c|c|c|c|c|c|c|c|}
$R_{\lambda} / N^{3}$ & $32^{3}$ & $48^{3}$ & $64^{3}$ & $96^{3}$ & $128^{3}$ & $192^{3}$ & $256^{3}$ & $384^{3}$ & $512^{3}$ \\
\hline 50 & 0.56 & 0.83 & $\underline{\mathbf{1 . 1 1}}$ & 1.67 & 2.22 & 3.34 & 4.45 & 6.67 & 8.90 \\
\hline 100 & 0.20 & 0.29 & 0.39 & 0.59 & 0.79 & $\underline{\mathbf{1 . 1 8}}$ & 1.57 & 2.36 & 3.15
\end{tabular}

Table 1. The value of $k_{\max } \eta$ associated with different resolutions. The Kolmogorov scales are $\eta=5.87 \cdot 10^{-3}$ and $\eta=2.07 \cdot 10^{-3}$ for $R_{\lambda}=50$ and $R_{\lambda}=100$, respectively.

A first, global assessment of the resolution that is achieved may be inferred by evaluating the product of the cut-off wavenumber and the observed Kolmogorov dissipation length-scale $\eta=L\left(3 R_{\lambda}^{2} / 20\right)^{-3 / 4}$ in terms of the Taylor Reynolds number $R_{\lambda}$ computed for the initial condition (see (33) for the definition) and integral length $L=1 / 2$. In order to resolve all dynamically relevant length-scales, including the dissipation length-scale it is required that $k_{\max } \eta$ is sufficiently large. A commonly accepted criterion of adequate spatial resolution is that $k_{\max } \eta>1$. When the focus is on higher-order statistics, it is preferred to use larger values $\left(k_{\max } \eta>3 / 2\right)[11,46]$. In Table 1 the values of 
$k_{\max } \eta$ are presented for the two computational Reynolds numbers considered $R e=1060.7$ and $R e=4242.6$ which correspond to $R_{\lambda}=50$ and $R_{\lambda}=100$. We observe that in the first case a resolution of at least $64^{3}$ is required to achieve full resolution, while in the second case the minimal required resolution moves up to $192^{3}$.

For validation of the code, the flow was simulated for more than two eddyturnover times and a number of quantities were monitored:

$$
\begin{array}{rlrl}
\text { Total energy : } & \widehat{E}(t)=\sum_{\mathbf{k}} E(\mathbf{k}, t) & \\
\text { Taylor microscale : } & \lambda(t)=\left(5 \widehat{E}(t) / \sum_{\mathbf{k}} k^{2} E(\mathbf{k}, t)\right)^{1 / 2} \\
\text { Taylor Reynolds : } & R_{\lambda}(t)=\lambda(t) u(t) / \nu ; u(t)=\sqrt{\frac{2}{3} \widehat{E}(t)} \\
\text { Longitudinal skewness : } & S_{1}(t)=-\frac{\left\langle\left(\partial v_{1}(\mathbf{x}, t) / \partial x_{1}\right)^{3}\right\rangle}{\left\langle\left(\partial v_{1}(\mathbf{x}, t) / \partial x_{1}\right)^{2}\right\rangle^{3 / 2}}
\end{array}
$$

The operator $\langle\cdot\rangle$ in (34) refers to volume averaging.
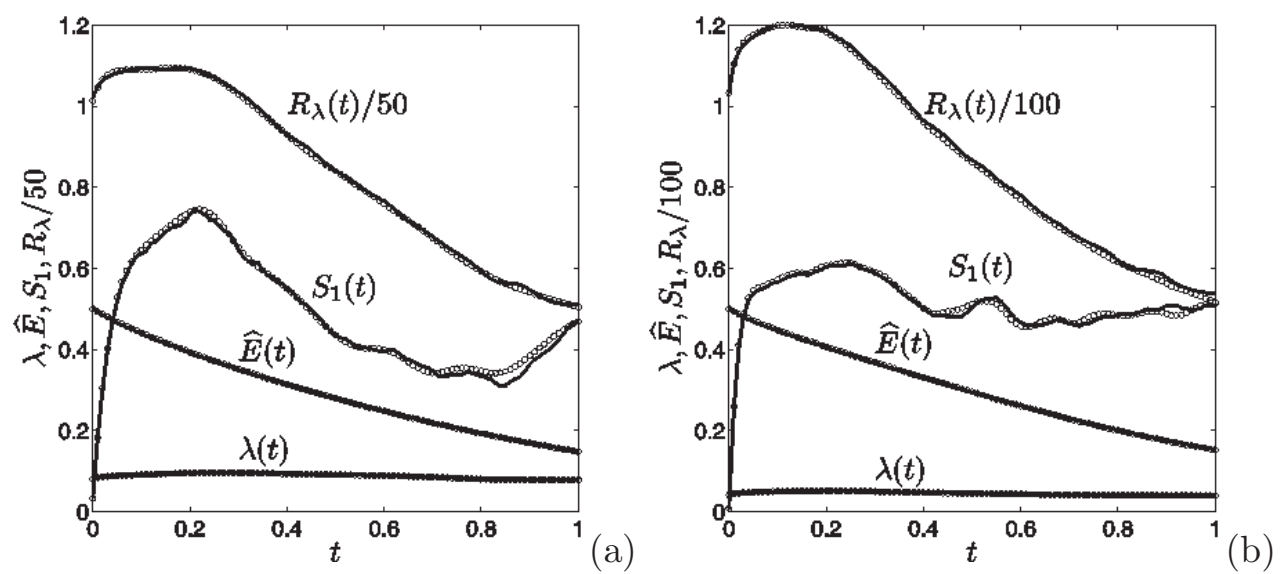

Figure 3. Prediction of total energy $\widehat{E}$, Taylor microscale $\lambda$, Taylor Reynolds number $R_{\lambda}$ and longitudinal skewness $S_{1}$ at an initial $R_{\lambda}=50$ (a) and $R_{\lambda}=100$ (b) with a finite-volume [35] (solid) and the present pseudo-spectral (dotted) code.

In Fig. 3] a comparison is made between simulation results obtained with the pseudo-spectral method at $N=512$, and with the high-order finite-volume discretization method [35]. For each of the quantities an almost perfect agreement may be observed. In Fig. 4 we assessed the convergence of the predictions as function of the spatial resolution. In this figure we replaced the longitudinal 

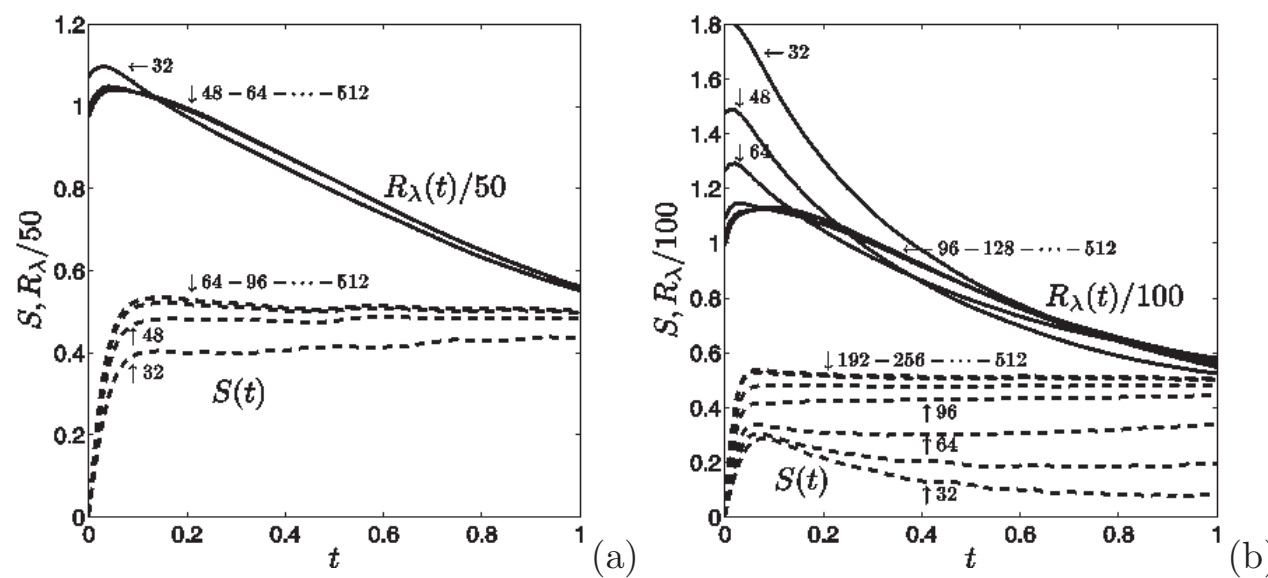

Figure 4. Convergence of predictions for the Taylor-Reynolds number $R_{\lambda}$ and the skewness $S$ at an initial $R_{\lambda}=50$ (a) and $R_{\lambda}=100$ (b) for different resolutions $N=32 \cdot 48 \cdot 64 \cdot 96 \cdot 128 \cdot 192 \cdot 256 \cdot 384 \cdot 512$.

skewness $S_{1}$ by the skewness:

$$
S(t)=\frac{2}{35}\left(\frac{\lambda(t)}{u(t)}\right)^{3} \sum_{\mathbf{k}} k^{2} T(\mathbf{k}, t)
$$

For homogeneous isotropic turbulence the value of $S$ should be equal to $0.5[2]$, which is quite well approximated in the simulations. This quantity is quite sensitive to the spatial resolution and is therefore a good indicator of appropriate spatial resolution. We observe that the different predictions display a clearly distinguishable convergence toward the grid-independent solution. Specifically, results obtained for resolutions higher than $64^{3}$ at $R_{\lambda}=50$ and $192^{3}$ at $R_{\lambda}=100$ are quite indistinguishable, consistent with the criterion that $k_{\max } \eta>1$.

In the next section we turn to the effects that different multiscale forcing procedures have on the developing turbulent flow. We will focus in particular on the modifications that arise in the kinetic energy spectrum.

\section{Modulated cascading by broad-band forcing}

The explicit forcing in different wavenumber bands can have a strong effect on the developing turbulent flow. We discuss the modifications of the energy spectrum arising from 'constant-energy' (class 'A') or 'constant-energy-inputrate' (class ' $\mathrm{B}$ ') procedures. The various forcing strategies will be shown to qualitatively correspond to each other, provided the total dissipation-rate $\varepsilon_{w}$ and the spectral energy distribution are commensurate for the different class 
' $\mathrm{A}$ ' and 'B' forcing strategies. We will specify this inter-relation in more detail momentarily. As point of reference, we will first turn our attention to forcing of the large scales only. Subsequently, we consider two-band forcing and investigate in particular the effects of variation of the strength and location of the small-scales band on the developing flow.

In the sequel, we consider time-averaged properties of the developing turbulent flow defined by:

$$
\langle h\rangle_{t}=\lim _{t \rightarrow \infty} \frac{1}{t-t_{0}} \int_{t_{0}}^{t} h(\tau) d \tau \approx \frac{1}{\mathcal{T}-t_{0}} \int_{t_{0}}^{\mathcal{T}} h(\tau) d \tau
$$

where $\mathcal{T}$ is sufficiently large. In all cases $t_{0}=5$ in order to allow the averagingprocess to start from a properly developed quasi-stationary state. The averaging is continued up to $\mathcal{T}=25$, which corresponds to approximately 40 eddy-turnover times. This was found to provide an accurate representation of the long-time averages, leading to relative errors below 5 percent, measured in terms of the ratio of the standard deviation and the mean signal. This procedure was applied to obtain the time-averaged kinetic energy spectra as well, which are very effective for monitoring changes in the kinetic energy dynamics due to the forcing.

Large-scale forcing. To create a point of reference, we first consider forced turbulence in which energy is introduced to the system only in the first shell $\mathbb{K}_{1,1}$. We adopt $k_{0}=3 \pi$ referring to Fig. 2(a) and force all 18 modes inside this band. The computational Reynolds number $R e=1060.7$ and the size of the computational domain $L_{b}=1$. The spatial resolution was taken to be $128^{3}$, which provides ample resolution of these cases, similar to what was established in subsection 2.3

In order to be able to quantitatively compare results obtained with the different forcing strategies, care should be taken of properly 'assigning' a level for the energy dissipation-rate and the spectral energy distribution. For this purpose, we may consider simulations with the A2-method to be central in the sense that the other three forcing strategies may be specified with reference to it. In fact, if we generate an initial condition with a certain total kinetic energy, then A2-forcing yields an evolving flow which becomes statistically stationary after some time, while maintaining the same level of total energy. The A2forced simulation can be used to specify the 'corresponding' class-B forcing strategies. In fact, the constant dissipation-rate $\varepsilon_{w}$ in class ' $\mathrm{B}$ ' forcing is taken equal to the time-average value of the dissipation-rate that is found from the A2-forced simulation, i.e., we adopt $\varepsilon_{w}=\langle\widehat{\varepsilon}\rangle_{t}$. This procedure was adhered to 
in all cases presented in this section. Finally, in the developed stages of either these A2- or B-forced flows, any instantaneous solution may be used to arrive at a full specification of the 'corresponding' A1-forcing. The actual choice of this instantaneous solution is arbitrary. However, when comparing simulations based on A1-forcing that adopt different realizations of the turbulent flow-field, we observed that the statistical properties of all these A1-forced cases were the same.
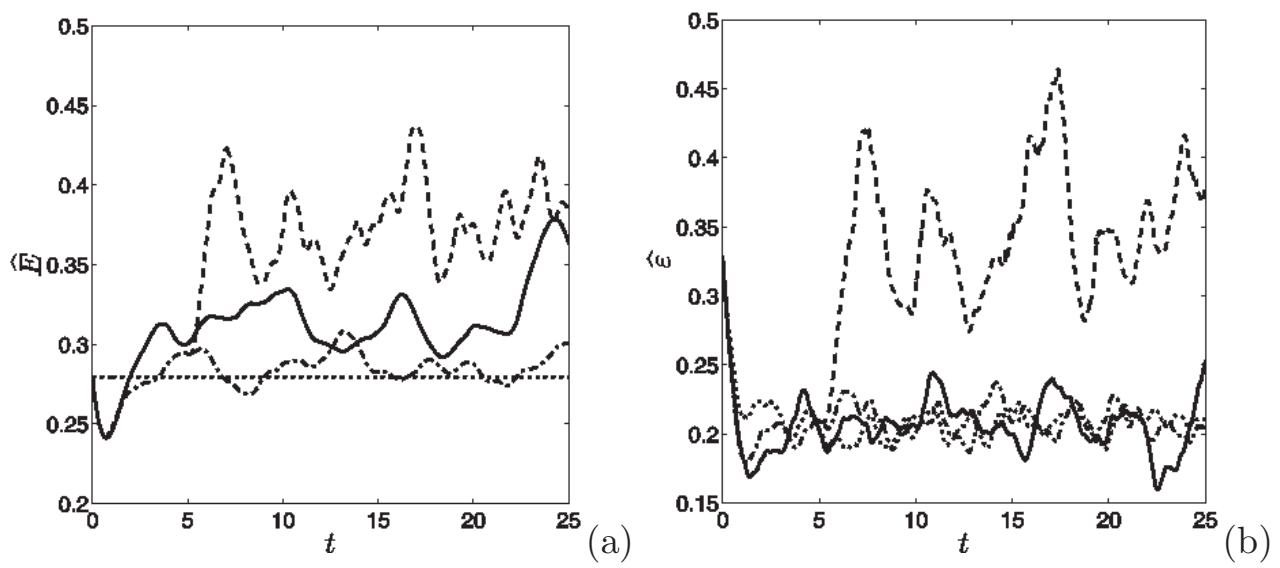

Figure 5. The evolution of the total kinetic energy $\widehat{E}$ (a) and energy-dissipation-rate $\widehat{\varepsilon}(\mathrm{b})$ for the large-scale forcing: A1 (dashed), A2 (dotted), B1 (dash-dotted), B2 (solid).

The evolution of the total kinetic energy $\widehat{E}(t)$ and energy dissipation rate $\widehat{\varepsilon}(t)$ is shown in Fig. 5. As initial condition for the A2- and B-forced simulations, we adopted the velocity field obtained at $t=0.5$ from the decaying homogeneous turbulence simulation discussed in subsection 2.3. To be able to qualitatively compare with the A1-forced flow at a similar energetic level we took as initial condition the solution from B1-forcing at $t=5$. The total kinetic energy is seen to fluctuate around its long-time mean value (of course, apart from A2-forcing). As can be seen, the system rapidly develops into a statistically stationary state characterized by the input of energy, its transfer to smaller scales and dissipation in the viscous range. In A1-forcing the Fourier-coefficients in the forced band are all kept constant, i.e., equal to their initial values. The energy in the system fluctuates very significantly, which was considered a disadvantage of this forcing in [11]. The energy and dissipation levels in A1-forcing differ considerably from those obtained with the other forcing strategies. To compare A2-forcing with B-forcing, the energy dissipation rate was taken as $\varepsilon_{w}=\langle\varepsilon\rangle_{t} \cong$ 0.2. The total kinetic energy for B1-forcing is seen to fluctuate around the constant value associated with A2-forcing. A similar impression is observed when use is made of the fractal B2-forcing in which the fractal dimension of 
the stirrer was taken equal to $D_{f}=2.6$ [32] which corresponds to an exponent $\beta=3 / 5$ in (25).

In general, when applied to the largest scales only, all forcing procedures mentioned in subsection 2.2 yield similar results. As a further example, the tails of the time-averaged spectra were found to be virtually identical to each other, which indicates that the properties of the smaller turbulent length-scales are not very strongly dependent on the details of the specific forcing. This was also established by various other quantities that were investigated. Specifically, the Taylor-Reynolds number $R_{\lambda}$ for the simulated cases was seen to fluctuate in the range between $\approx 50$ up to $\approx 60$ for all methods. The time-averaged value of the skewness was also investigated and found to be very close to 0.5 . This indicates that a well developed isotropic flow was attained [2].

Two-band forcing. In the simulations that adopt two-band forcing we consider situations in which we introduce energy into the system in a band consisting of four shells, next to the already described large-scale forcing in the first shell. We first compare the different class ' $A$ ' and ' $B$ ' forcing strategies, within this two-band setting. As second, forced band we consider $\mathbb{K}_{17,20}$. This band corresponds to $k_{1}=33 \pi$ and $k_{2}=41 \pi$ in Fig. 2(a) and contains in total 17284 different modes that are all explicitly forced. The comparison of the different forcing strategies shows that the flow-predictions are qualitatively comparable. Subsequently, we therefore focus on the B2-forcing strategy and investigate the effects arising from changes in the strength or the location of the second forced band.

Forcing of a second band implies that we need to additionally specify how the energy input is distributed over the bands, the shells within the bands and, finally, the modes within the shells. The specification of the A2-forcing requires the fraction of the energy-input that is allocated to the different bands. We consider the case in which $a=1 / 5$ in (28) which corresponds to equipartitioning of the energy-input over the five shells that are forced. The forcing within the second band is further specified by assigning an equal energy-input rate to each of the four shells contained in it. Finally, each of the modes in a particular shell $n$ receives an equal share of the energy-input to that shell, taking the number $P_{n}$ of modes in the particular shell into account. To compare the ' $\mathrm{A}$ ' with ' $\mathrm{B}$ ' forcing strategies we adopt the same method as above for specifying the parameter $\varepsilon_{w}$. Specifically, the total energy injection for the ' $\mathrm{B}$ ' methods was given as $\varepsilon_{w}=\langle\widehat{\varepsilon}\rangle_{t} \cong 1$, in terms of the time-average of the total dissipation rate in the A2-forcing. Moreover, the same equi-partitioning of the energy-input as in A2-forcing was adopted. Finally, the A1-forcing is derived from the field that was obtained at $t=5$ with the B1-forcing. We verified that the statistical properties of the A1-forced flow are insensitive to 
the particular choice of initial field used to define this forcing method.
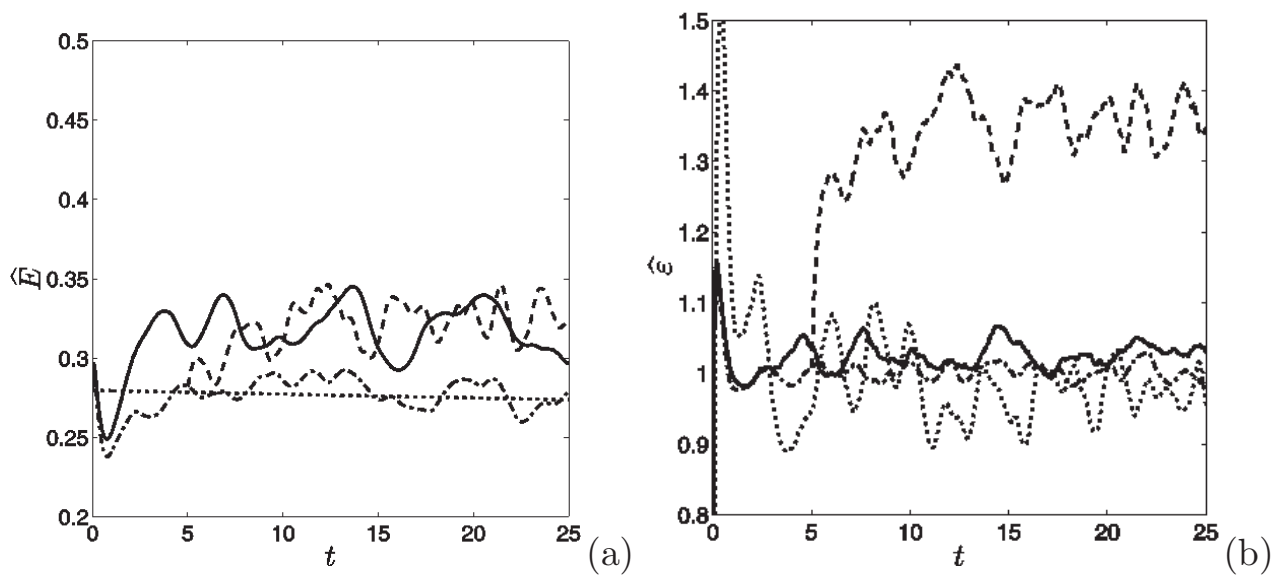

Figure 6. The evolution of the total kinetic energy $\widehat{E}$ (a) and energy-dissipation-rate $\widehat{\varepsilon}(\mathrm{b})$ for two-band forcing: A1 (dashed), A2 (dotted), B1 (dash-dotted), B2 (solid).

As may be noticed by comparing Fig. [5] with Fig. [6] the two-band forcing leads to a strong increase in the total energy dissipation-rate, while the total kinetic energy present in the flow is quite unaffected by the second forced band. The increase in the dissipation-rate is particularly strong for A1-forcing. Hence, the high- $k$ forcing changes mainly the distribution of energy over the scales and not so much the actual energy content. By changing the strength and location of the forcing, we have the possibility to control, and to some extent manipulate the way the energy is distributed and hence indirectly influence the large- and small-scale transport properties of the flow. We turn to this aspect next, by focusing explicitly on the kinetic energy spectrum.

The compensated kinetic energy spectra $E(k)=\langle\widehat{\varepsilon}\rangle_{t}^{-2 / 3} k^{5 / 3}\left\langle E_{k}\right\rangle_{t}$ that are obtained with the different two-band forcing methods are collected in Fig. 7 The modifications in the spectrum, relative to the case of large-scale forcing only, are localized primarily in the region close to the forced band. All forcing methods are seen to yield qualitatively quite similar results. Next to the expected modifications near the explicitly forced band, we observe that the two-band forcing also affects a much wider set of larger scale modes. In fact, a significant depletion of the kinetic energy in a range of scales 'ahead of' the forced region, is readily appreciated. This indicates that the agitation of a small band of modes can induce large changes in a rather wide part of the spectrum which further characterizes the type of turbulence-control that one may achieve with explicit forcing.

We next turn to the second part of this section and consider the effects of varying the spectral support and the strength of the second forced band. 


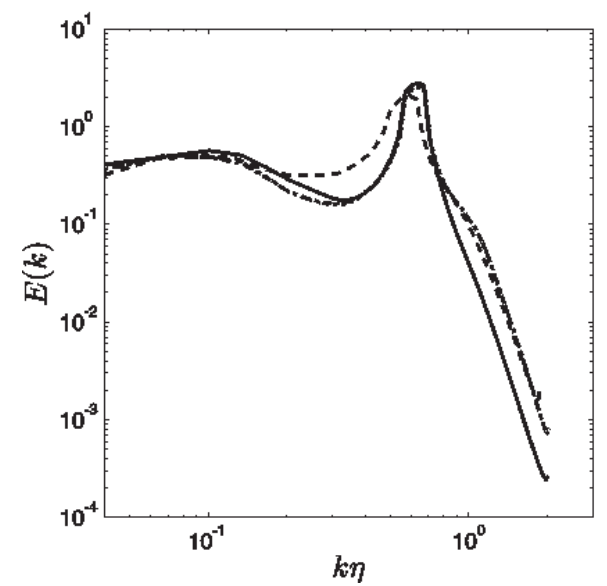

Figure 7. Compensated energy spectrum for two-band forced turbulence $(k \leq 3 \pi$ and $33 \pi<k \leq 41 \pi)$ with different methods: A1 (dashed), A2 (dotted), B1 (dash-dotted), B2 (solid).

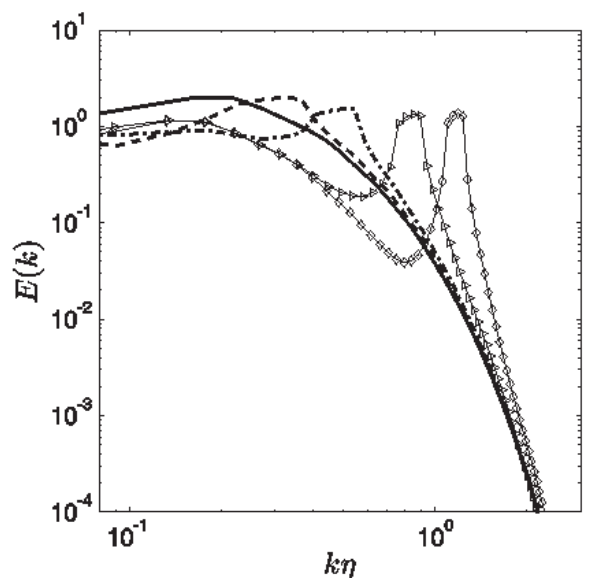

Figure 8. Compensated energy spectrum for two-band forced turbulence with the B2-method and the same energy inputs $\varepsilon_{w, 1}=\varepsilon_{w, 2}=0.15$ to the $k \leq 3 \pi$ band and various locations of the second band: $9 \pi<k \leq 17 \pi, 17 \pi<k \leq 23 \pi, 33 \pi<k \leq 41 \pi, 49 \pi<k \leq 57 \pi$ (dashed, dash-dotted, $\triangleright, \diamond)$.

The spectrum obtained with large-scale forcing at $\varepsilon_{w}=0.15$ in $k \leq 3 \pi$ band (solid).

The qualitative similarity of the different two-band forcing methods as seen in Fig. 7 allows to concentrate on only one of the forcing methods. We adopt B2-forcing in the sequel. In Fig. 8 we illustrate the effect of variation of the spectral support of the second band. Relative to the case of large-scale forcing only, we observe that the tails of the spectra are quite unaffected. However, the injection of energy in the second band is seen not only to increase the energy in the forced scales but also to deplete the energy in all the larger scales. Moreover, the 'up-scale' effect of energy-depletion is more pronounced in case the second band is moved toward smaller scales. 

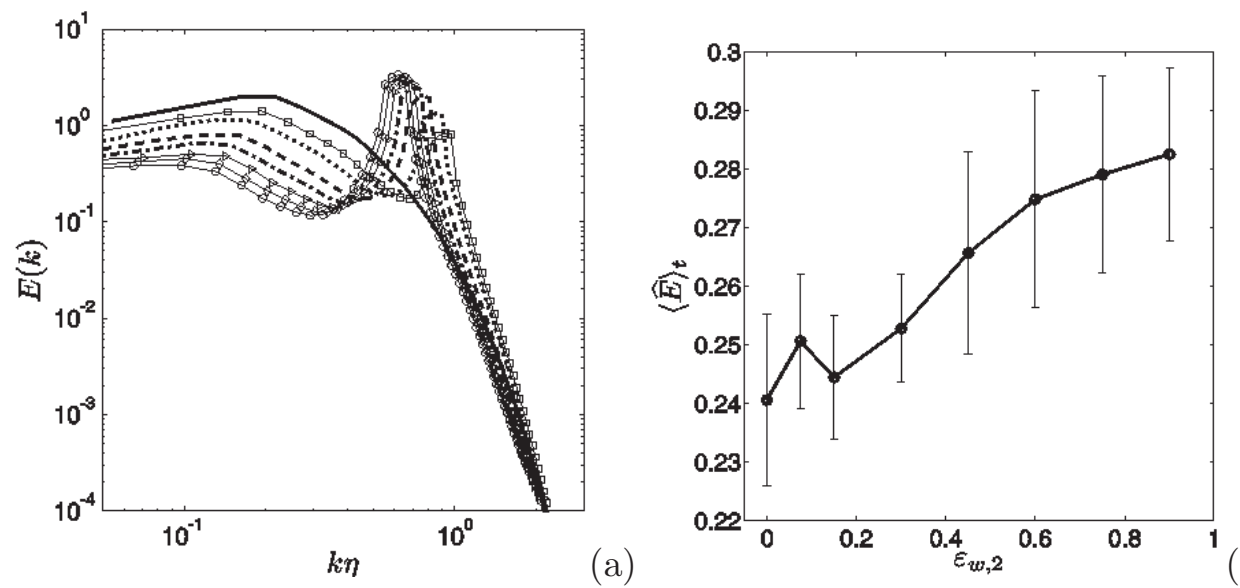

Figure 9. (a) Compensated energy spectrum for two-band forced turbulence with the B2-forcing method and energy input rate $\varepsilon_{w, 1}=0.15$ in $k \leq 3 \pi$ band for different strengths of forcing in the $33 \pi<k<41 \pi$ band: $\varepsilon_{w, 2}=0.075,0.15,0.30,0.45,0.60,0.75,0.90$ ( $\square$, dotted, dashed, dash-dotted,

$\triangleright, \diamond, \circ)$. Large-scale forcing with $\varepsilon_{w}=0.15$ in the $k \leq 3 \pi$ band is denoted by the solid line. (b)

Corresponding time-averaged total kinetic energy with standard deviations.

The control over the flow that is available with two-band forcing is examined further by investigating the effects of varying the strength of the forcing in the second band. We kept the energy input rate for the first $k \leq 3 \pi$ band equal to $\varepsilon_{w}=0.15$ and varied the strength of forcing in the second $33 \pi<k \leq 41 \pi$ band adopting $\varepsilon_{w}=0.075 \ldots 0.90$. The corresponding compensated energy spectra from these simulations are shown in Fig. 9(a). We observe that a higher energy input-rate in the second band leads to a more pronounced peak in the spectrum which shifts to lower values of $k \eta$ with increasing $\varepsilon_{w}$ of the second band. Simultaneously, the value of $E(k)$ decreases for the low- $k$ modes with increasing $\varepsilon_{w}$. This forcing of the second band allows to quite independently control the spectrum, at roughly the same total energy content in the flow. In fact, variation of $\varepsilon_{w}$ of the second band by a factor of about 10 is seen to lead to a comparably strong increase in the peak value of the spectrum while the total energy level $\langle\widehat{E}\rangle_{t}$ is increased by only $\approx 15 \%$ as seen in Fig. Q(b).

In the next section we will examine how the changes in the flow properties due to the two-band forcing in spectral space influence the physical space mixing efficiency of a passive scalar.

\section{Small and large scale mixing efficiency}

The consequences of explicit broad band forcing not only express themselves in modulated energy cascades. The mixing properties of the evolving turbulent flow in physical space also depend significantly on the forcing that is applied. 
In this section we quantify the mixing-efficiency by monitoring geometric properties of evolving level-sets of an embedded passive scalar. The numerical integration method that is used to determine these level-set properties is described in subsection 4.1. The ensemble-averaged simulation results are discussed in subsection 4.2 we establish to what extent the two-band forcing can be used to control the maximal rate of mixing and the total accumulated degree of mixing.

\subsection{Level-set evaluation to quantify mixing}

To illustrate and quantify the influence of two-band forcing on the turbulent dispersion of a passive scalar field we analyze the evolution of basic geometric properties of its level-sets. As a result of the turbulent flow these levelsets become highly distorted and dispersed across the flow-domain. Specifically, we concentrate on the surface-area and the wrinkling of these level-sets. We adopt a specialized integration method to determine these geometric properties, as developed in [14]. This method is based on the Laplace transform and avoids the explicit construction and integration over the complex and possibly fragmented scalar level-sets. With this method an accurate and efficient evaluation of the evolving mixing efficiency can be achieved which allows to quantify the increased complexity of the flow in relation to the two-band forcing that is used.

Basic geometric properties of a level-set $S(a, t)=\{\mathbf{x} \in \mathbb{R} \mid C(\mathbf{x}, t)=a\}$ of the scalar $C(\mathbf{x}, t)$ may be evaluated by integrating a corresponding 'density function' $g$ over this set. In fact, we have:

$$
I_{g}(a, t)=\int_{S(a, t)} d A g(\mathbf{x}, t)=\int_{V} d \mathbf{x} \delta(C(\mathbf{x}, t)-a)|\nabla C(\mathbf{x}, t)| g(\mathbf{x}, t)
$$

where the volume $V$ encloses the level-set $S(a, t)$ [31]. Setting $g(\mathbf{x}, t)=1$, $g(\mathbf{x}, t)=\nabla \cdot \mathbf{n}(\mathbf{x}, t)$ or $g(\mathbf{x}, t)=|\nabla \cdot \mathbf{n}(\mathbf{x}, \mathbf{t})|$, we can determine the global surface-area, curvature or wrinkling of $S(a, t)$ respectively. Here $\mathbf{n}(\mathbf{x}, t)=\nabla C(\mathbf{x}, t) /|\nabla C(\mathbf{x}, t)|$ is a unit normal vector, locally perpendicular to the level-set. The divergence of this vector-field is directly related to the local curvature of the level-set.

We will focus on the evolution of the surface-area $A$ and the wrinkling $W$. The scalar $C$ is scaled to be between 0 and 1 ; we will primarily consider the level-set $a=1 / 4$. In particular we monitor:

$$
\vartheta_{A}(a, t)=\frac{I_{A}(a, t)}{I_{A}(a, 0)} \quad ; \quad \vartheta_{W}(a, t)=\frac{I_{W}(a, t)}{I_{W}(a, 0)}
$$


By determining $\vartheta_{A}$ and $\vartheta_{W}$ we may quantify the rate at which surface-area and wrinkling develop, the maximal values that are obtained and the timescale at which these are achieved. The corresponding cumulative effects are given by

$$
\zeta_{A}(a, t)=\int_{0}^{t} \vartheta_{A}(a, \tau) d \tau \quad ; \quad \zeta_{W}(a, t)=\int_{0}^{t} \vartheta_{W}(a, \tau) d \tau
$$

These cumulative measures express the total surface-area and wrinkling that has developed in the course of time. In particular applications, e.g., involving combustion in diffusion flames, the cumulative surface-area and wrinkling express the total 'chemical processing capacity'. Here, we will determine these cumulative effects in order to characterize the different two-band forcing procedures.

To establish the influence of forcing on turbulent mixing properties we simulated the spreading of a passive tracer at Schmidt number $S c=0.7$. The simulations were started from a spherical tracer distribution of radius $r=3 / 16$. The scalar concentration was set equal to 1 inside this sphere and 0 outside. A localized Gaussian smoothing of this $C$-distribution was applied near the edge of the initial sphere to avoid resolution problems. The fractal forcing B2procedure as defined in (25) was adopted. We performed numerical simulations in which the energy input-rate $\varepsilon_{w}$ and the spectral support of this two-band forcing were varied.

As point of reference we adopted large-scale forcing in the $\mathbb{K}_{1,1}$ shell with an energy injection-rate $\varepsilon_{w}=0.6$. The resolution requirements were satisfactorily fulfilled: $k_{\max } \eta$ ranges from 2.3 to 3.5 using a resolution in the range $128^{3}-192^{3}$ grid-cells. For the passive scalar these resolutions correspond to $k_{\max } \eta_{O C}$ in the range from 3 to 4.5 , where $\eta_{O C}$ is the Obukhov-Corrsin scale [47]. To study the influence of two-band B2-forcing we applied supplementary forcing either in a region situated near the largest scales of the flow, i.e., $\mathbb{K}_{5,8}$ or further separated, i.e., $\mathbb{K}_{13,16}$. In case two bands are forced, the energy input-rate for the $\mathbb{K}_{1,1}$ shell is $\varepsilon_{w, 1}=0.15$, while the second band is forced using $\varepsilon_{w, 2}=0.45$. In this way the total energy level is kept at comparable levels in the different cases. A qualitative impression of the effect of these forcing procedures may be observed from the snapshots shown in Fig. 10. The velocity and passive scalar display considerably more small-scale features in case of two-band forcing, particularly in case of high- $k$ forcing. To quantify this qualitative impression we apply the level-set analysis discussed above. The results will be presented in the next subsection. 

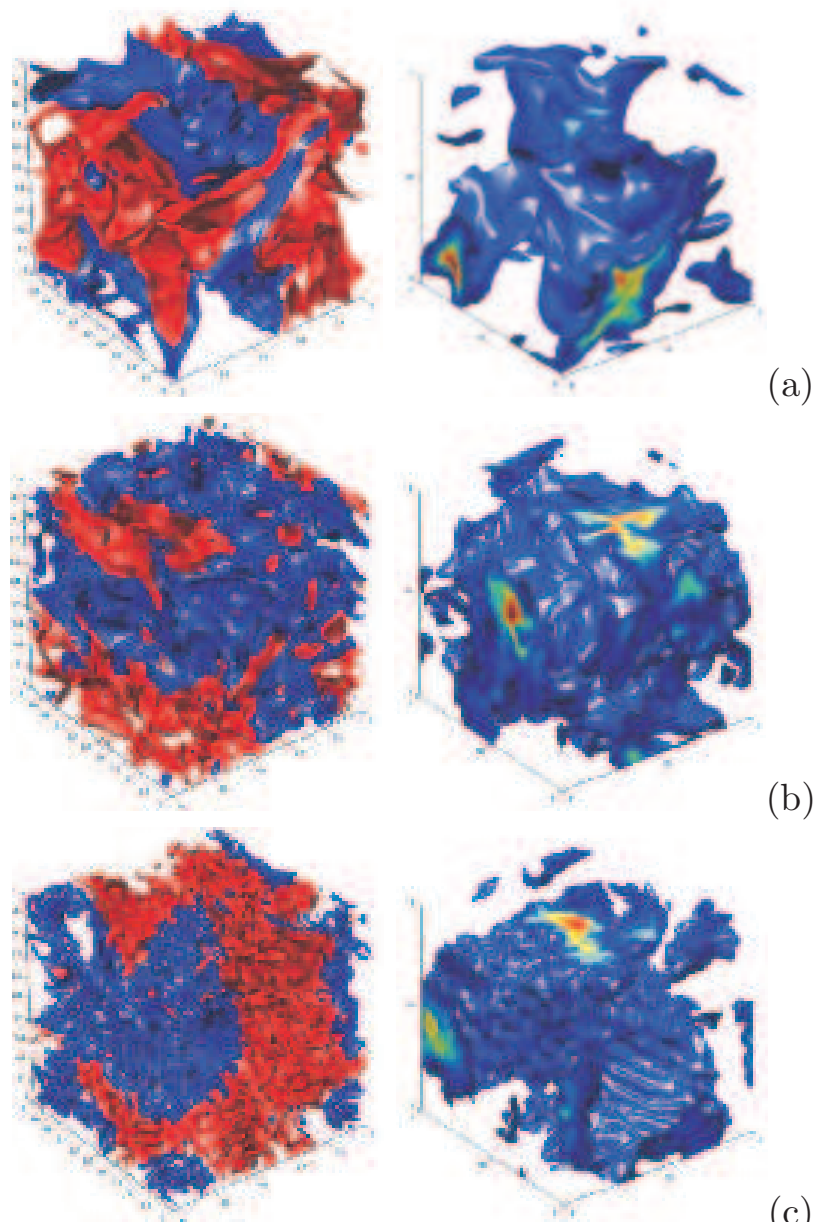

(c)

Figure 10. Snapshot of vertical velocity field iso-surfaces (left) and passive scalar concentration (right) at $t=0.5$ for large-scale forcing $\mathbb{K}_{1,1}$ with $\varepsilon_{w}=0.6$ (a), or with $\varepsilon_{w, 1}=0.15$ in the first shell and complementary forcing $\varepsilon_{w, 2}=0.45$ in $\mathbb{K}_{5,8}$ (b) or $\mathbb{K}_{13,16}$ (c). In the velocity field snapshots the red iso-surface corresponds to $u_{2}=0.2$ and the blue iso-surfaces to $u_{2}=-0.2$. The iso-surface at $C=0.25$ is shown for the passive scalar.

\section{$4.2 \quad$ Surface-area and wrinkling}

In this subsection, we compare instantaneous and accumulated mixing properties for large-scale forcing and different two-band forcing. The total energy input rate to the flow is kept constant at 0.6 ; a fraction $\varepsilon_{w, 1}$ is allocated to the first shell and $\varepsilon_{w, 2}$ to the second band such that $\varepsilon_{w, 1}+\varepsilon_{w, 2}=0.6$ and $\varepsilon_{w, 1}$ is varied from 0.05 up to 0.6 . The characterization of the mixing-efficiency was based on averaging 20 simulations, each starting from an independent realization of the initial velocity field. The different initial conditions were each 

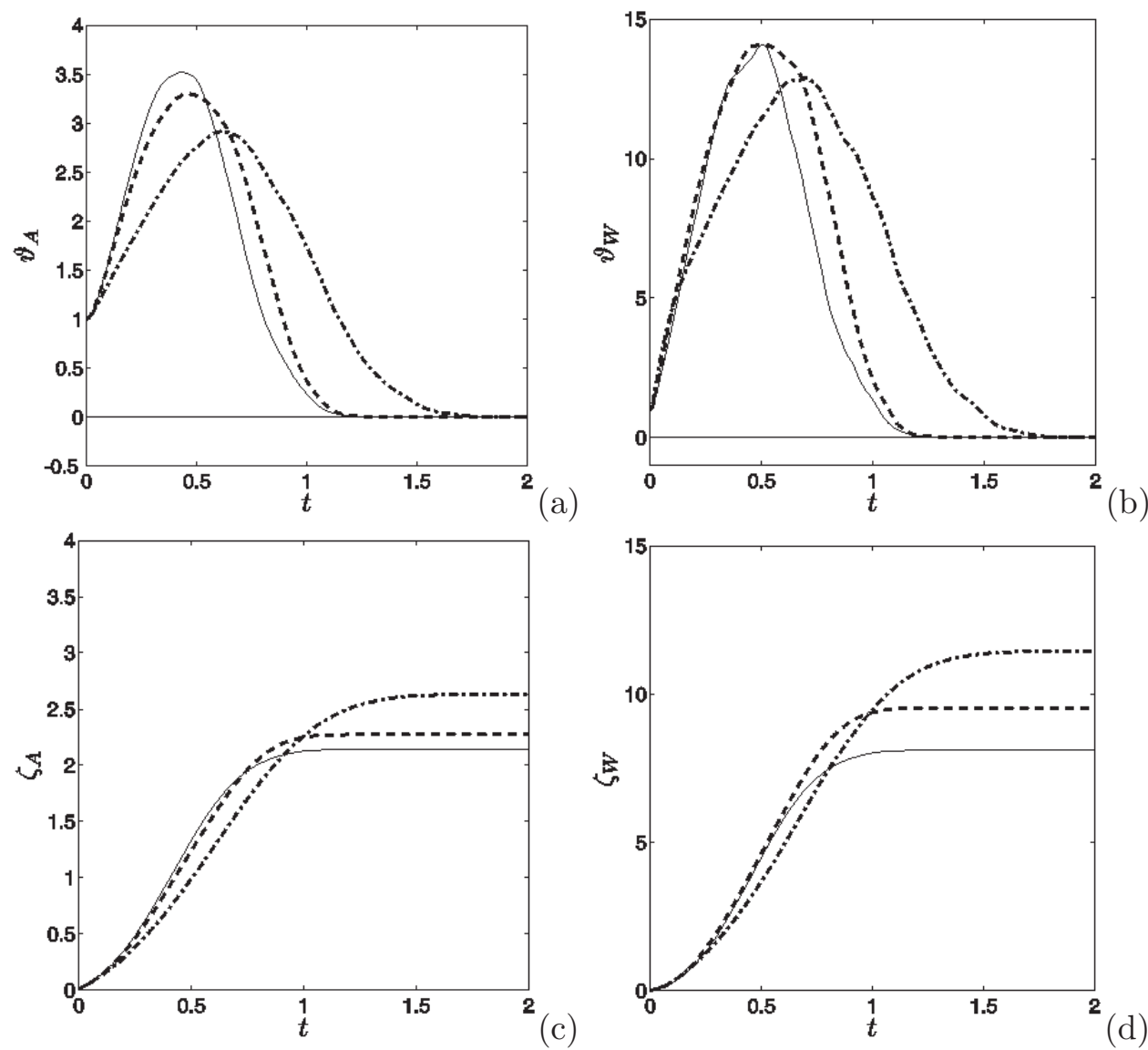

Figure 11. Evolution of decaying passive scalar growth parameters: a) surface-area $\vartheta_{A}$, b) wrinkling $\vartheta_{W}$, c) accumulated surface-area $\zeta_{A}$, d) accumulated wrinkling $\zeta_{W}$. Large-scale forcing $\mathbb{K}_{1,1}$ with $\varepsilon_{w}=0.60$ (solid) and complementary two-band forcing $\left(\varepsilon_{w, 1}=0.15\right.$ and $\left.\varepsilon_{w, 2}=0.45\right)$ in $\mathbb{K}_{5,8}$ (dashed), $\mathbb{K}_{13,16}$ (dash-dotted).

separated by two eddy-turnover times.

The instantaneous and cumulative effects arising from both the large-scale and the two-band forcing are shown in Fig. 11] The development of the instantaneous surface-area and wrinkling is qualitatively similar in each case. The concentrated initial tracer distribution is in the first stages primarily dispersed by convective sweeping in the turbulent flow. As a result, the level-set corresponding to $a=1 / 4$ becomes distorted and both $\vartheta_{A}$ and $\vartheta_{W}$ show a rapid increase. However, since no source of scalar was included in the computational model, molecular diffusion dominates the long-time behavior and leads to $\vartheta_{A}$ and $\vartheta_{W}$ to decrease to zero as $t \rightarrow \infty$. In between, $\vartheta_{A}$ and $\vartheta_{W}$ reach their maximum. The rapid initial growth is also clearly expressed in Fig. 11](c-d). In addition, the cumulative measures $\zeta_{A}$ and $\zeta_{W}$ show a clear saturation as 

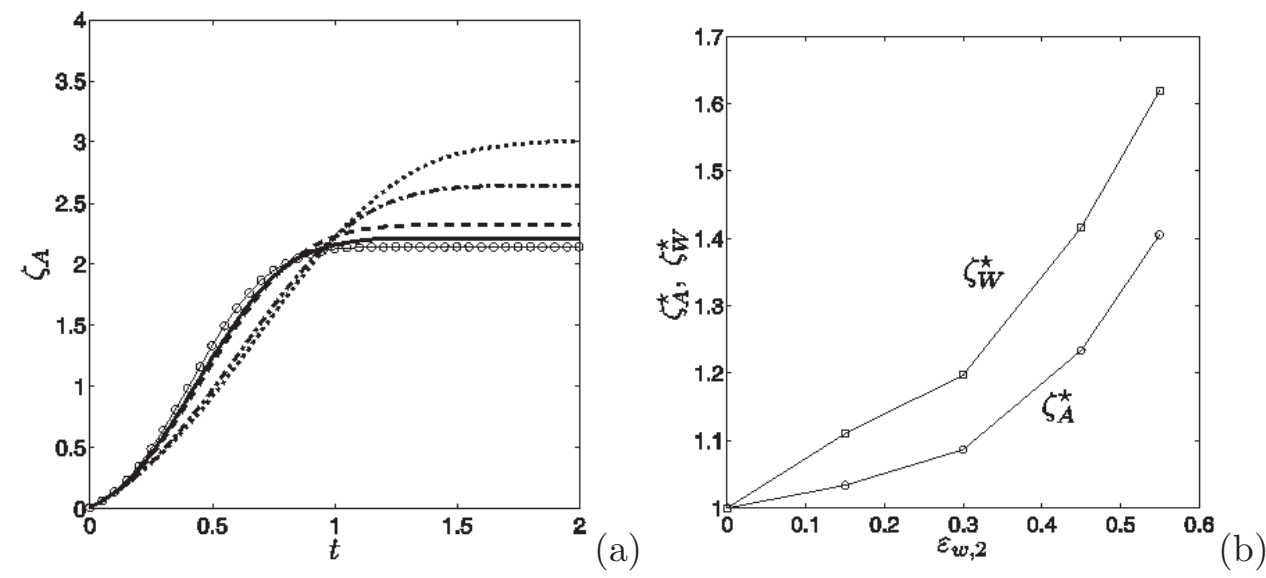

Figure 12. (a) Evolution of the decaying passive scalar accumulated surface-area parameter $\zeta_{A}$ for two-band forcing $\left(\mathbb{K}_{1,1}-\mathbb{K}_{13,16}\right)$ with different equi-partitions of energy between bands: $(0.60-0.00)(0),(0.45-0.15)($ solid $),(0.30-0.30)$ (dashed), $(0.15-0.45)$ (dot-dashed),

$(0.05-0.55)$ (dotted). (b) Total surface-area $\zeta_{A}^{\star}$ and wrinkling $\zeta_{W}^{\star}$ at $t=2$ for different $\varepsilon_{w, 2}$ in

$\mathbb{K}_{13,16}$ (results normalized by the total surface-area and wrinkling for the large-scale forcing).

$t \gtrsim 1$.

We observe from Fig. 111(a-b) that forcing of the large scales only, creates the highest growth-rate of surface-area and wrinkling. The surface-area reaches its maximum value both sooner and at a higher value in this case. In the initial stages convective spreading of the tracer dominates over the decay caused by molecular diffusion; hence in these stages the agitation of the larger scales plays a crucial role in the evolution of the surface-area. The higher band forcing needs to compete more directly with the viscous effects and does not induce very strong sweeping motions over large distances. Correspondingly, high- $k$ forcing is found less effective in producing surface-area. The more localized distortions of the scalar level-sets, as expressed by the development of the wrinkling, are less affected by the competition with viscosity, as seen in Fig. 111(b).

The interpretation of the effectiveness of the mixing in relation to the specific forcing alters if we compare the accumulated values for surface-area and wrinkling. As may be appreciated from Fig.11(c-d), a significant enhancement of the accumulated long-time surface-area and wrinkling arises as a result of the explicit agitation of the smaller scales in the flow. Evidently, forcing of the smaller scales does not yield a more intense mixing, judging from the instantaneous values, but does yield an increase in the total surface-area and wrinkling, accumulated over time.

To measure the influence of variations in the strength of the forcing in the high- $k$ band, we focus on the $\mathbb{K}_{1,1}$ and $\mathbb{K}_{13,16}$ two-band forcing. In particular, we keep $\varepsilon_{w, 1}+\varepsilon_{w, 2}=0.6$ and vary the values of $\varepsilon_{w, 2}$. The effects on the cumulative mixing-efficiency are shown in Fig. 12(a). We observe that an 
increase in $\varepsilon_{w, 2}$ implies a slight decrease in the initial growth rate of instantaneous surface-area, but an increase in the long-time cumulative effect. The dependence of the long-time cumulative effect on $\varepsilon_{w, 2}$ is clarified in Fig. 12(b). These simulation results establish the degree of control that may be achieved with two-band forcing and the feasibility of such computational modeling. This approach may help to identify optimal stirring procedures to which future research will be devoted.

\section{Summary and concluding remarks}

Various deterministic forcing methods that perturb a turbulent flow in a chosen range of length-scales were examined. The presented modeling framework incorporates the explicit forcing as an integral part. We have shown that with a relatively simple forcing model basic properties of complex flows can be captured. For example, an enhancement of the energy dissipation by smallscale forcing was seen to produce so-called spectral short-cut features, quite similar to what was observed experimentally in flow over canopies [12] where the kinetic energy is immediately transferred to the smallest scales of the flow.

Forcing methods agitating the flow in a wide range of scales induce significant differences in the developing flow, compared to the case obtained classically in which only the large scales are forced. Various forcing methods were introduced and shown to produce qualitatively similar results, provided the forcing parameters correspond to turbulence at comparable total kinetic energies. We classified the methods according to constant-energy or constant-energyinput-rate and examined these procedures by simulating forced turbulence with energy injected in two different bands. The modulation of the turbulent flow was investigated for various locations of the second high- $k$ band in spectral space. It was shown that the forcing in the second band induces nonlocal modulation of the energy spectrum. This was further examined by simulations done with different strength of forcing in the high- $k$ band controlled by the energy injection rate.

We devoted special attention to a recently proposed multiscale forcing that models a flow under the influence of an additional perturbation by a multiscale object [32]. We performed numerical simulations of the dispersion of a passive scalar field in a turbulent flow that is driven by such forcing. A level-set integration method was adopted to quantify general characteristics of mixing quality and efficiency. It was found that broad-band forcing causes additional production of smaller scales in the flow which is directly responsible for the localized enhancement of the wrinkling of the level-set. In contrast, the surface-area of a level-set of the tracer is found to be mainly governed by convective sweeping by the larger scales in the flow and hence it is governed to a greater extent by 
the energy input-rate allocated to the small- $k$ range. Future study will include the spatial localization of the forcing. This can help to model flows that are more closely related to realistic physical situation observed in experiments and applications.

\section{Acknowledgments}

The work is supported by the Foundation for Fundamental Research on Matter (FOM), Utrecht, the Netherlands. Simulations were performed at SARA; special thanks go to Willem Vermin for support with the parallelization. The computations were made possible through grant SC-213 of the Dutch National Computing Foundation (NCF). AKK would like to thank David McComb for many stimulating discussions during research visits at the University of Edinburgh. BJG gratefully acknowledges support from the Turbulence Working Group (TWG) at the Center for Non-Linear Studies (CNLS) at Los Alamos National Laboratory which facilitated an extended research visit in 2005 and allowed many fruitful discussions. Special thanks go to Darryl Holm.

\section{Appendix A:}

To eradicate the aliasing error we study in more detail (i) the random phase shifts method and (ii) the method employing two-shifted grids with spherical truncation $[6,40,41]$. In the first case, the aliasing error is only partially removed. With additional truncation of the Fourier velocity field coefficients the remaining error can be reduced to $\mathcal{O}\left(\Delta t^{2}\right)$. In the method employing twoshifted grids and spherical truncation, the aliasing error can be fully removed from the simulations. This specific approach doubles the computational costs and memory requirements, compared to the random shifts method. The wellknown $3 / 2$ method can be used as well, by going to higher resolution and truncating the field. This can be done with the lowest number of operations, but has higher memory requirements.

The aliasing error for higher-resolution runs affects mainly the small-scale statistics. This is visualized in Fig. A1 where we have shown the TaylorReynolds number and the longitudinal skewness for decaying turbulence simulations with initial $R_{\lambda}=100$ and two resolutions $128^{3}$ and $192^{3}$. The partial dealiasing removes the main aliasing error and with the additional truncation reduces it to the accuracy associated with the adopted Runge-Kutta scheme. There is a small difference between the full and partial removal of the aliasing error for the lower resolution of $128^{3}$, but this largely vanishes for the wellresolved $192^{3}$ case. 

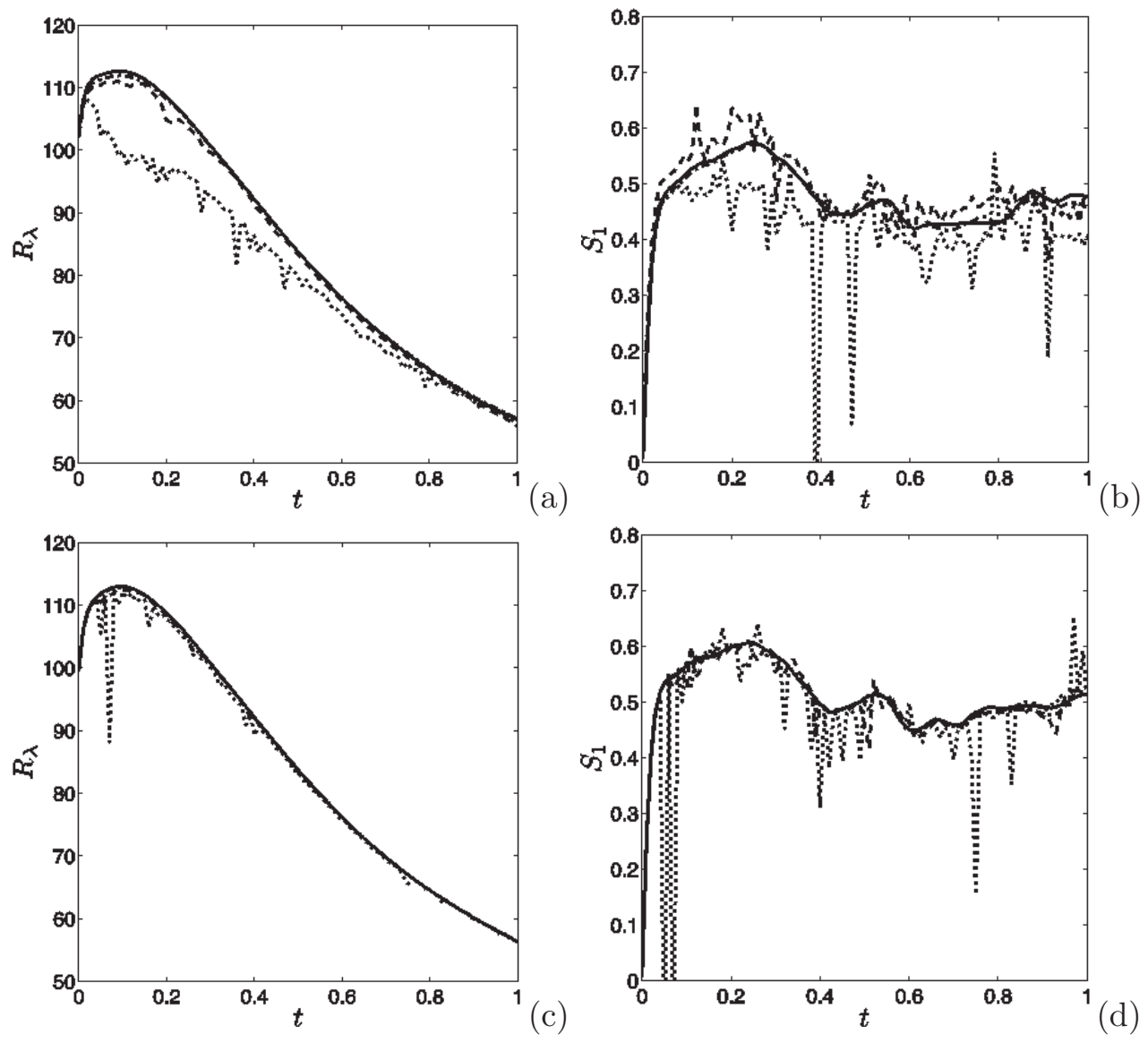

Figure A1. Influence of aliasing error for resolution $128^{3}$ (a-b) and $192^{3}$ (c-d) on Taylor-Reynolds number $R_{\lambda}(t)$ and longitudinal skewness $S_{1}(t)$ at an initial $R_{\lambda}=100$ case. Simulations with aliasing error (dotted), partial dealiasing without truncation (dashed), partial dealiasing with the truncation (dash-dotted), full dealiasing by two grid shifts (solid). Results for the partial dealiasing with truncation (dash-dotted) are almost identical to fully dealiased results (solid).

\section{References}

[1] J. Banhart, M.F. Ashby, and N.A. Fleck. Cellular metals and metal foaming technology. Verlag MIT, Bremen, 2001.

[2] G. K. Batchelor. Theory of homogeneous turbulence. Cambridge University Press, 1953.

[3] K. Boomsma, D. Poulikakos, and F. Zwick. Metal foams as compact high performance heat exchangers. Mech. Mater., 35:1161-1176, 2003.

[4] W. P. Breugem and B. J. Boersma. Direct numerical simulations of turbulent flow over a permeable wall using a direct and a continuum approach. Phys. Fluids, 17, 2005.

[5] W. P. Breugem and D. A. S. Rees. A derivation of the volume-averaged Boussinesq equations for flow in porous media with viscous dissipation. Transp. Porous Media, to appear.

[6] C. Canuto, M. Hussaini, A. Quarteroni, and T. Zang. Spectral Methods in Fluid Dynamics. Springer Verlag (Berlin and New York), 1988.

[7] D. Carati, S. Ghosal, and P. Moin. On the representation of backscatter in dynamic localization models. Phys. Fluids, 7(3):606-616, 1995.

[8] J. R. Chasnov. Simulation of the Kolmogorov inertial subrange using an improved subgrid model. Phys. Fluids, 3(1):188-200, 1991. 
[9] S. Chen and X. Shan. High-resolution turbulent simulations using the Connection Machine-2. Comput. Phys., 6(6):643-646, 1992.

[10] H. Cheng and I. P. Castro. Near wall flow over urban-like roughness. Boundary-Layer Meteorology, 104(2):229-259, 2002.

[11] V. Eswaran and S. B. Pope. An examination of forcing in direct numerical simulations of turbulence. Comput. Fluids, 16:257, 1988.

[12] J. Finnigan. Turbulence in plant canopies. Ann. Rev. Fluid Mech., 32:519-571, 2000.

[13] M. Frigo and S. G. Johnson. FFTW: An adaptive software architecture for the FFT. In Proc. 1998 IEEE Int. Conf. Acoustics Speech and Signal Processing, volume 3, pages 1381-1384, http://www.fftw.org, 1998.

[14] B. J. Geurts. Mixing efficiency in turbulent shear layers. J. Turbul., 2:2-24, 2001.

[15] B. J. Geurts. Modern Simulation Strategies for Turbulent Flow. R.T. Edwards, 2001.

[16] S. Ghosal, T. S. Lund, P. Moin, and K. Akselvoll. A dynamic localization model for large-eddy simulation of turbulent flows. J. Fluid Mech., 286:229-255, 1995.

[17] Silicon Graphics. SCSL user's guide. TechPubs Library of Silicon Graphics, http://techpubs.sgi.com.

[18] S. Grossman and D. Lohse. Scaling in hard turbulent Rayleight-Beńard flow. Phys. Rev. A, 46(2):903-917, 1992.

[19] HDF5 Group. HDF5 user's guide. National Center for Supercomputing Applications (NCSA), University of Illinois, http://www.hdfgroup.org.

[20] J. O. Hinze. Turbulence: An Introduction to its Mechanism and Theory. New York, McGrawHill, 1975.

[21] T. Ishihara and Y. Kaneda. High resolution DNS of incompressible homogeneous forced turbulence - time dependence of statistics. In Y. Kaneda and T. Gotoh, editors, Statistical Theories and Computational Approaches to Turbulence. Springer, 2003.

[22] J. Jimenez. Turbulent flow over rough walls. Ann. Rev. Fluid Mech., 36:173-196, 2004.

[23] J. Jimenez, A. A. Wray, P. G. Saffman, and R. S. Rogallo. The structure of intense vorticity in isotropic turbulence. J. Fluid Mech., 255:65-90, 1993.

[24] Y. Kaneda, T. Ishihara, M. Yokokawa, K. Itakura, and A. Uno. Energy dissipation rate and energy spectrum in high resolution direct numerical simulations of turbulence in a periodic box. Phys. Fluids, 15(2):21-24, 2003.

[25] R. M. Kerr. Higher-order derivative correlations and the alignment of small-scale structures in isotropic numerical turbulence. J. Fluid Mech., 153:31-58, 1985.

[26] R. M. Kerr. Velocity, scalar and transfer spectra in numerical turbulence. J. Fluid Mech., 211:309-332, 1990.

[27] A. N. Kolmogorov. The local structure of turbulence in incompressible viscous fluids at very large Reynolds numbers. C.R. Acad. Sci. URSS, 30:301-305, 1941.

[28] A. N. Kolmogorov. A refinement of previous hypothesis concerning the local structure of turbulence in a viscous incompressible fluid at high reynolds number. J. Fluid Mech., 13:82-85, 1962.

[29] F. Li, L. Lefferts, and T. H. van der Meer. Study on heat transfer enhancement by metallic foams with carbon nano fibers (CNFs). In Proceedings of the 6th World Conference on Experimental Heat Transfer, Fluid Mechanics, and Thermodynamics, Matsushima, Miyagi, Japan, April 17$21,2005$.

[30] L. Machiels. Predictability of small-scale motion in isotropic fluid turbulence. Phys. Rev. Lett., 79(18):3411-3414, 1997.

[31] V.G. Maz'ja. Sobolev spaces. Springer Verlag - Berling, 1985.

[32] B. Mazzi and J. C. Vassilicos. Fractal generated turbulence. J. Fluid Mech., 502:65-87, 2004.

[33] W. D. McComb. The Physics of Fluid Turbulence. Oxford University Press, 1990.

[34] J. Meyers. Accuracy of Large-Eddy Simulation strategies. PhD thesis, Katholieke Universiteit Leuven, 2004.

[35] J. Meyers, B. J. Geurts, and M. Baelmans. Database analysis of errors in large-eddy simulation. Phys. Fluids, 15(9):2740-2755, 2003.

[36] A. Misra and D. I. Pullin. A vortex-based subgrid stress model for large-eddy simulation. Phys. Fluids, 9(7):2443-2454, 1997.

[37] K. Mohseni, B. Kosovic, S. Shkoller, and J. E. Marsden. Numerical simulations of the Lagrangian Averaged Navier-Stokes equations for homogeneous isotropic turbulence. Phys. Fluids, 15(2):524-544, 2003

[38] MPI. Message Passing Interface standard. http://www.mpi-forum.org.

[39] S. B. Pope. Turbulent Flows. Cambridge University Press, 2000. 
[40] R. S. Rogallo. An ILLIAC program for the numerical simulation of homogeneous incompressible turbulence. Technical Report NASA-TM-73203, NASA, 1977.

[41] R. S. Rogallo. Numerical experiments in homogeneous turbulence. Technical Report NASA-TM81315, NASA, 1981.

[42] SARA. Computing and Networking Services. http://www.sara.nl.

[43] E. D. Siggia and G. S. Patterson. Intermittency effects in a numerical simulation of stationary three-dimensional turbulence. J. Fluid Mech., 86:567, 1978.

[44] A. A. Townsend. The Structure of Turbulent Shear Flows. Cambridge Univ. Press, 1976.

[45] A. Vincent and M. Meneguzzi. The spatial structure and statistical properties of homogeneous turbulence. J. Fluid Mech., 225:1-20, 1991.

[46] L. P. Wang, S. Chen, J. G. Brasseur, and J. C. Wyngaard. Examination of hypotheses in the Kolmogorov refined turbulence theory through high-resolution simulations. Part 1. Velocity field. J. Fluid Mech., 309:113-156, 1996.

[47] T. Watanabe and T. Gotoh. Statistics of a passive scalar in homogeneous turbulence. New J. Phys., 6(40):1-36, 2004.

[48] P. Wesseling. An introduction to multigrid methods. Wiley, New York, 1992.

[49] S. Whitaker. The Forchheimer equation: a theoretical development. Transp. Porous Media, 25:27-61, 1996.

[50] Y. Yamazaki, T. Ishihara, and Y. Kaneda. Effects of wavenumber truncation on high-resolution direct numerical simulation of turbulence. J. Phys. Soc. Jpn., 71(3):777-781, 2002.

[51] P. K. Yeung and J. G. Brasseur. The response of isotropic turbulence to isotropic and anisotropic forcing at the large scales. Phys. Fluids A, 3(5):884-897, 1991.

[52] A. J. Young. Investigation of Renormalization Group Methods for the Numerical Simulation of Isotropic Turbulence. Phd thesis, University of Edinburgh, 1999. 